\begin{tabular}{|c|c|}
\hline Title & Selective eradication of pluripotent stem cells by inhibiting DHODH activity \\
\hline Author(s) & Kondo, Toru \\
\hline Citation & $\begin{array}{l}\text { Stem cells, 39(1), 33-42 } \\
\text { https://doi.org/10.1002/stem.3290 }\end{array}$ \\
\hline Issue Date & 2021-01 \\
\hline Doc URL & http:/hdl.handle.net/2115/82146 \\
\hline Rights & $\begin{array}{l}\text { Kondo, T. Selective eradication of pluripotent stem cells by inhibiting DHODH activity. Stem Cells. 2021; 39: 33-42. } \\
\text { https://doi.org/10.1002/stem.3290. @ } 2020 \text { A l phaM ed Press }\end{array}$ \\
\hline Type & article (author version) \\
\hline File Information & SC-20-0166.R1_Proof_hi.pdf \\
\hline
\end{tabular}

Instructions for use 


\title{
Selective eradication of pluripotent stem cells by inhibiting DHODH activity
}

Toru Kondo ${ }^{\mathrm{a}}$

aDivision of Stem Cell Biology, Institute for Genetic Medicine, Hokkaido University, Sapporo, Hokkaido 060-0815, Japan

Correspondence: Toru Kondo, Ph.D.

Division of Stem Cell Biology, Institute for Genetic Medicine, Hokkaido University, Kita-15, Nishi-7, Kita-ku, Sapporo, Hokkaido 060-0815, Japan

Tel: +81-11-706-6082, Fax: +81-11-706-7870, E-mail: tkondo@igm.hokudai.ac.jp

Running head: Selective elimination of PSCs by BRQ

Author contributions: Toru Kondo: conception and design, financial support, collection and assembly of data, data analysis and interpretation, manuscript writing, final approval of manuscript.

Key words: pluripotent stem cells (PSCs), dihydroorotate dehydrogenase (DHODH), brequinar (BRQ), teratoma, regenerative medicine, cell therapy

\begin{abstract}
Pluripotent stem cells (PSCs), such as embryonic stem cells and induced pluripotent stem cells, give rise to all kinds of functional cells, making them promising for
\end{abstract}


successful application in regenerative medicine. However, there is concern that a PSCderived differentiated cell population may form teratomas when used for cell therapy if the population contains undifferentiated PSCs. Therefore, for the success of regenerative medicine, it is crucial to establish methods that induce complete PSC differentiation and eliminate the contamination of PSCs. Here, I show that the dihydroorotate dehydrogenase (DHODH) inhibitor brequinar (BRQ) induced cell cycle arrest, cell death, and stemness loss in PSCs, whereas it was less toxic against normal tissue-specific stem cells and differentiating cells. I demonstrate that BRQ-pretreated PSCs did not form teratomas after being transplanted into NOD/SCID mice. Moreover, BRQ administration to teratoma-bearing mice prevented tumour growth and decreased PSC marker levels in the tumour without any visible effects in the differentiated germ layer cells and the mice. Collectively, these data indicated that DHODH inhibitors such as BRQ can be indispensable in the fundamental methods of PSC-based therapy.

\section{Significance statement}

For the success of regenerative medicine, it is crucial to establish methods that exclude the contamination of PSCs in the differentiated cell population. The results of this study show that BRQ, the strongest available inhibitor of DHODH, specifically targets PSCs, whereas it is less cytotoxic towards lineage-committed progenitor cells and differentiated functional cells. Furthermore, BRQ prevents teratoma formation in vivo without any visible side effects in mice. These findings identify DHODH inhibitors, such as BRQ, as fundamental reagents for PSC-based cell therapy.

\section{Introduction}


Pluripotent stem cells (PSCs), such as embryonic stem cells (ESCs) and induced pluripotent stem cells (iPSCs), proliferate indefinitely and give rise to all three functional germ layer cells, including neurons, muscle cells, and blood cells; therefore, PSCs have been extensively investigated for their characteristics and potential use in regenerative medicine. ${ }^{1-5}$ However, since undifferentiated PSCs form tumours called teratomas, which can contain all three germ layer cells when transplanted in vivo, the development of methods that induce complete cell differentiation, eliminate the contamination of PSCs, and/or prevent teratoma formation after therapy is crucial for the success of regenerative medicine.

Many strategies have been developed to eliminate the contamination of PSCs from differentiated cultures by targeting PSC-specific characteristics, including the cell surface membrane protein SSEA5, methionine depletion, and alkaline phosphatase activity. ${ }^{6-8}$ Using high-throughput chemical screening, Ben-David et al. identified new compounds that selectively kill PSCs..$^{9}$ Although these strategies eliminate undifferentiated PSCs in culture, these methods, except the method shown by BenDavid et al., have not been shown to eradicate the propagation of teratomas after cell transplantation.

It has been demonstrated that PSCs and cancer cells produce ATP mainly by glycolysis, while most somatic cells utilize oxidative phosphorylation to generate energy. ${ }^{10,11}$ The use of glycolysis is an advantage for PSCs because glycolysis does not generate harmful reactive oxygen species, which damage genomic and mitochondrial DNA and oxidize both proteins and lipids, but rather produces nucleotides and lipids, which support rapid PSC proliferation. Glutamine has been shown to be another major 
carbon source for the proliferation of both PSCs and cancer cells. ${ }^{12,13}$ These findings indicate that PSCs and cancer cells utilize the same metabolic pathways to proliferate.

There is increasing evidence that dihydroorotate dehydrogenase (DHODH), an essential enzyme for the de novo pyrimidine synthesis pathway, is an attractive therapeutic target for many types of cancer, including melanoma, acute myeloid leukaemia, breast cancer, pancreatic cancer, and glioblastoma. ${ }^{14-18}$ DHODH inhibitors not only induce cell cycle arrest or cell death in cancer cells but also abolish stemness by preventing the O-GlcNAcylation of stem cell factors, such as Sox2 and Oct4. Given that glutamine is the substrate for the de novo pyrimidine synthesis pathway, these results suggest that DHODH inhibitors can be used to eliminate undifferentiated PSCs that persist throughout the differentiation-induction process. I show here that the DHODH inhibitor brequinar (BRQ) eliminated undifferentiated PSCs but was less toxic to normal cells, such as neural stem cells (NSCs), astrocytes, and myoblasts, in culture. BRQ also induced nuclear export of the PSC factors Oct4 and Nanog in a chromosomal maintenance 1 (Crm1)-dependent manner. Moreover, administration of BRQ prevented PSC tumorigenesis in vivo without inducing any visible side effects in mice.

Collectively, these data indicate that DHODH inhibitors can be used in an essential basic technique of PSC therapy. 


\section{Materials and methods}

\section{Animals and chemical reagents}

All experiments with mice were performed according to protocols approved by the Animal Care and Use Committees of Hokkaido University. The mice were obtained from Hokudo, Inc. (Sapporo, Japan, www.hokudo.co.jp), and chemicals and growth factors were purchased from Invitrogen (Carlsbad, CA, www.lifetechnologies.com/nl/en/home/brands/invitrogen.html) and PeproTech (Rocky Hill, NJ, www.peprotech.com), respectively, unless otherwise indicated.

\section{Cell culture}

Mouse ESCs (E14tg2a, gift from Dr. Masui) and iPSCs (iPS-Hep-FB/Ng/gfp-103C-1, RIKEN BRCs, Tsukuba, Japan) were maintained on gelatine- or iMatix 511 (Takara Bio Inc, Shiga, Japan, www.takara.co.jp)-coated culture plates in Glasgow MEM (GMEM, Sigma-Aldrich, St. Louis, MO, www.sigmaaldrich.com) supplemented with chemicals, sodium pyruvate $(1 \mathrm{mM}), 1 \mathrm{x}$ nonessential amino acids, 2 -mercaptoethanol (0.1 mM, Nacalai Tesque, Kyoto, Japan), Leukemia nhibitory factor (LIF, ESGRO, 1000 units/ml, Merck Millipore, Darmstadt, Germany, www.merckmillipore.com), KnockOut Serum Replacement (KSR, 15\%, Thermo Fisher Scientific, Grand Island, New York), foetal calf serum (FCS, 1\%, HyClone), penicillin, and streptomycin (Nacalai Tesque, Kyoto, Japan) (PSC medium). ESCs were transfected with pEGFP-N1 (Clontech, Takara Bio Inc, Shiga, Japan, www.takara.co.jp) using Lipofectamine 3000 (Thermo Fisher Scientific, Grand Island, New York) according to the manufacturer's protocol and then selected in the 
presence of G418 (300 $\mu \mathrm{g} / \mathrm{ml}$, Nacalai Tesque, Kyoto, Japan), establishing EGFPexpressing ESCs (GFP ${ }^{+}$ESCs).

NSCs were prepared from an embryonic day 17.5 mouse telencephalon and cultured as a monolayer on poly-D-lysine (PDL, $15 \mu \mathrm{g} / \mathrm{ml})$ - and fibronectin $(1 \mu \mathrm{g} / \mathrm{ml}$, Invitrogen)-coated culture dishes in DMEM/F12 (Sigma-Aldrich, St. Louis, MO, www.sigmaaldrich.com) supplemented with chemicals, bFGF (10 ng/ml), and EGF (10 $\mathrm{ng} / \mathrm{ml}$ ) (NSC medium), as described previously. ${ }^{19}$ Astrocyte differentiation was induced by culturing NSCs in DMEM (Nacalai Tesque, Kyoto, Japan) containing FCS (10\%), penicillin, and streptomycin (FCS medium) for three weeks, as described previously. ${ }^{20}$ For cytotoxicity assays, PSCs were cultured on gelatine-coated 96-well plates in PSC medium, while NSCs and astrocytes were cultured on PDL- and fibronectin-coated 96well plates in NSC and FCS media, respectively.

C2C12 myoblasts (gift from Dr. Sabe) and the PA6 stromal cell line (RIKEN BRC, Tsukuba, Japan) ${ }^{21}$ were cultured in DMEM and aMEM (Nacalai Tesque, Kyoto, Japan), respectively, both of which were supplemented with FCS (10\%), penicillin, and streptomycin.

To examine cell cycle arrest, the MTT assay and BrdU incorporation assay were performed as described previously. ${ }^{22,23}$

To analyse cytotoxicity, annexin $\mathrm{V}$ immunostaining was performed using the ApoScreeen Annexin V-FITC Apoptosis Kit (Beckman Coulter, Brea, CA). Additionally, GFP-expressing PSCs $\left(5 \times 10^{5}\right.$ cells) were co-cultured with NSCs $\left(3 \times 10^{5}\right.$ cells), which were labelled using PKH26 Red Fluorescent Cell Linker Kits (SigmaAldrich, St. Louis, MO, www.sigmaaldrich.com), in the presence or absence of BRQ $(10 \mu \mathrm{M})$. Three days after incubation, cells were harvested using accutase (Nacalai 
Tesque, Kyoto, Japan) and suspended in $10 \mathrm{ml}$ of PBS. One microliter of the cell suspension was mixed with $9 \mu 1$ of trypan blue (Nacalai Tesque, Kyoto, Japan) and then, $\mathrm{GFP}^{+}$and $\mathrm{PKH} 26^{+}$cells were quantified using an AxioImager M1 microscope (Carl Zeiss, Oberkochen, Germany). The kits were used according to the manufacturer's protocol.

\section{PSC-derived NSC differentiation}

NSC differentiation from mouse PSCs was induced using RHB-A medium (Takara Bio Inc, Shiga, Japan, www.takara.co.jp) according to the supplier's instructions. In brief, mouse PSCs were cultured in RHB-A medium alone for 5 days on iMatrix 511-coated culture plates. The medium was replaced with RHB-A medium containing bFGF (10 $\mathrm{ng} / \mathrm{ml})$ and EGF $(10 \mathrm{ng} / \mathrm{ml})$ on day 6 and with NSC medium on day 12 . On day 15 , the cells were harvested and cultured as a monolayer on PDL- and fibronectin-coated culture dishes in NSC medium. The medium was changed every 2-3 days. To induce neural differentiation, the cells were cultured in 8-well chambers in mitogen-free NSC medium or DMEM+1\% FCS for 1 week (Supporting information Fig. 8A, 8B).

PSCs were co-cultured with PSC-NSCs in the presence or absence of BRQ (1 or $10 \mu \mathrm{M}$ ) for 3 days, and then, Nanog+ and Nestin+ cells were quantified using an AxioImager M1 microscope (Carl Zeiss, Oberkochen, Germany).

\section{Quantitative reverse-transcription polymerase chain reaction}

Total RNA was extracted from cells using the RNeasy kit (Qiagen, Hilden, Germany, http://www1.qiagen.com) and reverse-transcribed using the Transcriptor First Strand cDNA Synthesis Kit (Roche Applied Science, Basel, Switzerland, http://www.roche- 
applied-science.com) according to the supplier's instructions. Real-time polymerase chain reaction (PCR) was performed on a StepOnePlus instrument (Thermo Fisher Scientific, Grand Island, New York) using THUNDERBIRD SYBR qPCR Mix (Toyobo, Tokyo, Japan) according to the manufacturer's protocol. The cycle parameters were as follows: $15 \mathrm{~s}$ at $95^{\circ} \mathrm{C}$ and $30 \mathrm{~s}$ at $60^{\circ} \mathrm{C}$ for 40 cycles. All gene expression data were normalized to the housekeeping gene $18 \mathrm{~S}$ ribosomal RNA according to the $\Delta \Delta \mathrm{Ct}$ method. The oligonucleotide primer sequences were as follows: mouse sox2, forward 5'-TGAAGAAGGATAAGTACACGCT-3' and reverse 5'TCCTGCATCATGCTGTAGCTG-3'; mouse oct4, forward 5'CTGAAGCAGAAGAGGATCACC-3' and reverse 5'CCGCAGCTTACACATGTTCTT-3'; mouse nanog, forward 5'CTGATTCTTCTACCAGTCCCAA-3' and reverse 5'AGAGTTCTTGCATCTGCTGGA-3'; and 18S, forward 5'CGGACAGGATTGACAGATTG-3', and reverse 5'CAAATCGCTCCACCAACTAA-3'.

\section{Immunochemistry}

Tumours were sectioned at a thickness of $10 \mu \mathrm{m}$ and immunostained as described previously. ${ }^{24,25}$ The following antibodies were used for immunostaining: rabbit polyclonal anti-Sox2 (1:500, StemCell Technology, Vancouver, BC, Canada, http://www.stemcell.com), mouse monoclonal anti-Oct4 (1:200, Cell Signaling Tech, Danvers, MA), rabbit polyclonal anti-Nanog (1:200, Wako Pure Chemical, Osaka, Japan, wako-chem.co.jp/english), rat monoclonal anti-GFP (1:500, Nacalai Tesque, Kyoto, Japan), rabbit monoclonal anti-Ki67 (1:100, Thermo Fisher Scientific, Grand 
Island, New York), rabbit polyclonal anti-active Caspase 3 (1:1000, Cell Signaling Technology, Danvers, MA), mouse monoclonal anti-Nestin (1:200, Rat401, BD Biosciences, San Jose, CA, www.bd.com), mouse monoclonal anti- $\beta$ III tubulin (1:400, Sigma-Aldrich, St. Louis, MO, www.sigmaaldrich.com), rabbit polyclonal anti-smooth muscle actin (SMA, 1:200, Proteintech, Rosemont, IL), and rabbit polyclonal anti-alpha fetoprotein (AFP, 1:100, Proteintech, Rosemont, IL). Antibodies were detected with Alexa568-conjugated goat anti-rabbit IgG (1:500, Thermo Fisher Scientific, Grand Island, New York); Alexa488-conjugated goat anti-mouse, anti-rabbit, or anti-rat IgG (1:500, Thermo Fisher Scientific, Grand Island, New York); DyLight 649 donkey antirabbit (1:500, BioLegend, San Diego, CA) and goat anti-mouse IgG-Cy3 (1:500, Jackson ImmunoResearch, West Grove, PA, www.jacksonimmuno.com). Cells were counterstained with DAPI ( $1 \mu \mathrm{g} / \mathrm{ml}$, D523, DOJINDO, Kumamoto, Japan) to visualize nuclei. Fluorescence images were obtained using an AxioImager M1 microscope.

Immunostaining results for cultured cells were quantified by analysing at least two 8-chamber slides. Over 1000 cells were counted from each well, and statistical analyses were performed using Microsoft Excel. Similar results were obtained from three independent experiments, and representative results are shown in each figure.

\section{Vector construction}

Full-length mouse dhodh cDNA was amplified from mouse NSC cDNA using KOD Plus-Ver.2 polymerase (Toyobo, Osaka, Japan, www.toyobo.co.jp) according to the manufacturer's instructions. These cDNAs were inserted into the p3xFLAG CMV10 vector (Sigma-Aldrich, St. Louis, MO, www.sigmaaldrich.com) to produce p3xFLAG CMV10-mDHODH. The following oligonucleotide DNA primers were synthesized: for 
the full-length mouse dhodh, the 5' primer was 5'-

AAGAATTCAATGGCGTGGAGACA-3', and the 3' primer was 5'TTGGATCCTCACCTGCGGTGATC-3’.

To knock down mouse $d h o d h$, three short hairpin (sh) sequences were generated using InvivoGen's siRNA Wizard (http://www.sirnawizard.com/). These sh sequences were inserted into a psiRNA-h7SKhygro G1 expression vector (InvivoGen, San Diego, CA, www.invivogen.com) to produce psiRNA-h7SKhygro-mDHODHsh13. mDHODHsh expression cassettes were then inserted into pGL3-EGFP vectors to generate pGL3-EGFP-mDHODHsh1-3 (dhodh sh expression vectors). The knockdown efficiency of these vectors was analysed by Western blotting and immunostaining (Supporting information Fig. 3). The most efficient vectors dhodh shl and $s h 3$ were used for the knockdown experiments. The sh1 and sh3 target sequences for mouse dhodh were 5'-GGCTAGCTGTTCGAGTCATCT-3' and 5'GGAAGCTGTGGACGGACTCTA-3', respectively. The control sh target (egfp) sequence was 5'-GCAAGCTGACCCTGAAGTTCA-3'.

The nucleotide sequences of the cloned cDNAs were verified using the BigDye Terminator Kit version 3.1 (Thermo Fisher Scientific, Grand Island, New York) and ABI sequencer model 3130xl (Thermo Fisher Scientific, Grand Island, New York). Cells were transfected with the vectors using Lipofectamine 3000 (Thermo Fisher Scientific, Grand Island, New York) according to the manufacturer's protocol.

\section{Teratoma formation}

PSCs were cultured in the presence or absence of BRQ for 2 days. The surviving cells $\left(1 \times 10^{6}\right)$ were then suspended in $50 \mu 1$ of Matrigel (BD Biosciences, San Jose, CA, 
www.bd.com) and injected subcutaneously into the hips of NOD/SCID mice aged 5-8 weeks, which were anaesthetized with $10 \%$ pentobarbital before the transplantation procedures. Four weeks after injection, mice were sacrificed, and the tumours were collected, photographed, and measured as described previously. ${ }^{23}$

To examine the anti-teratoma activity of BRQ, mice bearing the subcutaneous tumour were intraperitoneally administered $200 \mu \mathrm{l}$ of PBS or $25 \mathrm{mg} / \mathrm{kg}$ BRQ (TOCRIS Bioscience, Bristol, UK) daily in $200 \mu 1$ of PBS. Five days after BRQ treatment, mice were sacrificed, and the tumours were collected, photographed, and processed for immunostaining as described previously. ${ }^{23}$

\section{Statistical analysis}

Student's $t$ test was used to compare two paired groups. $P$ values less than .05 (twosided) were considered significant.

\section{Results}

\section{The DHODH inhibitor BRQ specifically inhibited the proliferation of PSCs}

I first examined whether DHODH inhibitors eliminated PSCs. I cultured mouse ESCs and iPSCs in various concentrations of the established DHODH inhibitors teriflunomide, leflunomide, vidofludimus, and BRQ for 3 days and then examined cell viability by the MTT assay. As shown in Fig. 1A, BRQ at less than $25 \mu \mathrm{M}$ completely prevented the proliferation of ESCs and iPSCs, while other inhibitors produced similar effects at only $200 \mu \mathrm{M}$. The half maximal growth inhibitory concentration $\left(\mathrm{GI}_{50}\right)$ values of BRQ were 4.67 and $6.41 \mu \mathrm{M}$ for ESCs and iPSCs, respectively. I next investigated the cytotoxicity induced by these DHODH inhibitors in primary cultured brain cells 
(NSCs and astrocytes), C2C12 myoblasts, and PA6 stromal cells. None of the inhibitors induced obvious cytotoxicity in NSCs or astrocytes, even at $100 \mu \mathrm{M}$, although BRQ and vidofludimus slightly inhibited the proliferation of $\mathrm{C} 2 \mathrm{C} 12$ and PA6 cells in a dosedependent manner (Fig. 1B and 1C; Supporting information Fig. 1). The GI $\mathrm{I}_{50}$ of BRQ was $31.5 \mu \mathrm{M}$ and $115 \mu \mathrm{M}$ for the $\mathrm{C} 2 \mathrm{C} 12$ and PA6 cells, respectively. Together, these data indicated that BRQ significantly inhibited the proliferation of PSCs, while it induced low levels of cytotoxicity in proliferating lineage-committed cells.

To verify that BRQ induced cytotoxicity in PSCs by blocking the de novo pyrimidine synthesis pathway, I performed two rescue experiments using uridine, the substrate for the salvage pathway, and uridine diphosphate (UDP), a downstream product of the pyrimidine synthesis pathway (Supporting information Fig. 2A). I cultured ESCs and iPSCs in the presence of BRQ with various concentrations of nucleosides for 3 days and then examined their viability. The addition of uridine abrogated BRQ-induced cytotoxicity in a dose-dependent manner (Fig. 1D). The addition of cytidine slightly reduced the cytotoxicity, whereas the addition of the other nucleosides did not show any effect (Fig. 1D). I then cultured ESCs and iPSCs in the presence of BRQ with various concentrations of nucleotide diphosphates, intermediate substrates for nucleic acid synthesis, and found that UDP addition abrogated the cytotoxicity in a manner similar to uridine, whereas the addition of the other nucleotide diphosphates showed no significant effects (Fig. 1E). Together, these data revealed that BRQ inhibited PSC proliferation by blocking DHODH activity and that the cytotoxicity of BRQ can be eliminated by accelerating the salvage pathway.

\section{BRQ induced cell cycle arrest and cell death in PSCs}


To confirm that BRQ induced cell cycle arrest in PSCs, I performed a bromodeoxyuridine $(\mathrm{BrdU})$ incorporation assay. Two days after BRQ treatment, 16\% and 14\% of the BRQ-treated ESCs and iPSCs, respectively, were positive for BrdU, whereas $30 \%$ and $35 \%$ of the control ESCs and iPSCs, respectively, were positive for BrdU (Fig. 2A).

Since I observed many floating cells in the BRQ-containing PSC culture, I examined whether BRQ induced the death of PSCs. I cultured PSCs in the presence or absence of BRQ for 2 days and then immunolabeled the cells for cleaved caspase 3 (Casp3). While $8 \%$ and $5 \%$ of the control ESCs and iPSCs, respectively, were positive for Casp3, 12\% and 22\% of the BRQ-treated ESCs and iPSCs, respectively, were positive for Casp3 (Fig. 2B; Supporting information Fig. 2A). Using annexin V immunoreactivity, I confirmed that BRQ induced cytotoxicity in PSCs: $58 \%$ and $71 \%$ of the BRQ-treated ESCs and iPSCs, respectively, were positive for annexin V, whereas $4 \%$ of both control ESCs and iPSCs were positive for annexin V (Fig. 2C).

To further verify that BRQ eliminated PSCs, I examined the expression of the core PSC genes Sox2, Oct4 and Nanog in BRQ-treated PSCs. Two days after BRQ treatment, I observed decreased expression of these three genes in BRQ-treated cultured cells compared with that in control cells in culture (Fig. 2D). Moreover, immunostaining indicated that the number of cells expressing PSC genes significantly decreased in the BRQ-treated culture, whereas most of the control PSCs were positive for these factors (Supporting information Fig. 2B). Taken together, these data revealed that BRQ eradicated PSCs by inducing cell cycle arrest and cell death in culture.

Using DHODH-specific shRNAs (Supporting information Fig. 3), I examined whether BRQ-dependent cytotoxicity induced in PSCs was dependent on the inhibition 
of DHODH activity. Three days after transfection with the dhodh sh-expression vectors, I immunolabeled the cells for Ki67 and Casp3. I observed that the number of Ki67+/GFP+ proliferating PSCs significantly decreased, whereas the number of Casp3+/GFP+ dying cells increased (Fig. 3; Supporting information Fig. 4), a finding similar to that observed in the BRQ-treated cells. These data showed that the BRQdependent cytotoxicity was induced by inhibition of DHODH activity.

\section{The proliferation/survival of PSCs was largely dependent on the de novo pyrimidine synthesis pathway}

I examined why DHODH inhibitors effectively prevent PSC proliferation, despite pyrimidine synthesis being regulated by both the de novo and salvage pathways (Supporting information Fig. 5A). I first examined the expression of dhodh and uridinecytidine kinases (uck1 and uck2), key regulators of the salvage pathway, ${ }^{26}$ in NSCs, ESCs, iPSCs, PA6 cells, and C2C12 cells. Both dhodh and ucks were highly expressed in ESCs, iPSCs, PA6 cells, and C2C12 cells compared with their expression in NSCs; however, the dhodh/uck1 ratio was significantly higher in ESCs (2.65) and iPSCs (5.16) than in other cells (1 in NSCs, 0.85 in PA6 cells, and 1.09 in C2C12 cells) (Supporting information Fig. 5B, 5C), suggesting that ESCs and iPSCs depend on the de novo pathway to a greater extent than NSCs, PA6 cells, and C2C12 cells. In addition, the human protein atlas (https://www.proteinatlas.org) revealed that the $D H O D H / U C K 1$ ratio in all normal human tissues, except the liver (1.56), is relatively low (less than 0.65) (Supporting information Fig. 5D). The following cancer cell lines have a high DHODH/UCK1 ratio: K562 (1.35), NB4 (1.77), U937 (1.46), Daudi (1.89), and U698 (2.52) (Supporting information Fig. 5E). These data are consistent with the previous 
finding that BRQ prevented tumorigenesis of U937 cells more efficiently than that of THP1 cells (0.8). ${ }^{15}$ Furthermore, ucks-knockdown PSCs continued to proliferate (Supporting information Fig. 6C), although dhodh knockdown induced cell cycle arrest and cell death (Fig. 3; Supporting information Fig. 2A). Eventually, I succeeded in establishing $u c k 1$ - and $u c k 2$-double knockdown PSCs (Supporting information Fig. 6D). Thus, these findings indicated that PSC proliferation is largely dependent on the de novo pyrimidine synthesis pathway, acting by increasing dhodh expression.

\section{BRQ induced the nuclear export of PSC factors in a Crm1-dependent manner}

Since it was previously demonstrated that the DHODH inhibitor induced nuclear export of Sox 2 in glioblastoma-initiating cells, ${ }^{18}$ independent of its cytotoxicity, I examined whether BRQ induces similar phenomena in PSCs. Two days after BRQ treatment, the number of PSCs expressing either Oct4 (2\% in the ESCs and $0 \%$ in the iPSCs) or Nanog (6\% in the ESCs and $0 \%$ in the iPSCs) significantly decreased compared with that of DMSO-treated control ESCs (Oct4, 85\% and Nanog, 86\%) and iPSCs (Oct4, 94\% and Nanog, 93\%) (Supporting information Fig. 7A, 7B). The addition of the pancaspase inhibitor Z-VAD did not rescue the reduction in Nanog $(2 \%$ in the ESCs and $5 \%$ in the iPSCs) or Oct4 (5\% in the ESCs and 3\% in the iPSCs) in the BRQ-treated PSCs, although it slightly delayed BRQ-dependent cell death, suggesting that cell death and the decreased levels of Nanog and Oct4 were independent events in the BRQtreated cells (Supporting information Fig. 7A, 7B).

Since it was previously shown that leptomycin B (LMB), a specific inhibitor of Crm1, prevented the nuclear export of Sox2 in DHODH inhibitor-treated GICs, ${ }^{18} \mathrm{I}$ also examined whether the addition of LMB would lead to Nanog and Oct4 retention in the 
nuclei of BRQ-treated PSCs. Two days after culturing PSCs with BRQ or BRQ plus LMB, I found that LMB completely blocked the nuclear export of Nanog and Oct4 in the BRQ-treated PSCs (Supporting information Fig. 7C, 7D). Thus, these data indicated that BRQ excluded Nanog and Oct4 from the nuclei in a Crm1-dependent manner.

\section{BRQ specifically eliminated PSCs co-cultured with NSCs}

The ultimate goal of this study was to demonstrate that BRQ prevents teratoma formation by depleting undifferentiated PSCs that reside with differentiating/differentiated cells in vitro and in vivo. I therefore co-cultured PSCs with NSCs in the presence or absence of BRQ and then examined the specific cytotoxicity induced by BRQ in the PSCs. Three days after the co-culture was seeded, I immunolabeled the cells for Nanog and the NSC marker Nestin. In the cultures with 10 $\mu \mathrm{M}$ BRQ, no Nanog ${ }^{+}$cells (red in the ESCs, green in the iPSCs) were detected, although many surviving cells were Nestin ${ }^{+}$(green in the ESCs, red in the iPSCs) (Fig. 4A, 4B). In contrast, both $\mathrm{Nanog}^{+}$and $\mathrm{Nestin}^{+}$cells were observed in the cultures treated with DMSO alone or $1 \mu \mathrm{M}$ BRQ (Fig. 4A, 4B). To quantify the BRQ-dependent specific level of cytotoxicity induced in PSCs, I co-cultured GFP-expressing PSCs with PKH26-labelled NSCs in the presence and absence of $10 \mu \mathrm{M}$ BRQ for 3 days and then counted the number of surviving cells. In the absence of BRQ, the numbers of GFP ESCs and PKH26 $6^{+}$NSCs were $170 \times 10^{5}$ cells and $12 \times 10^{5}$ cells, respectively, while those of $\mathrm{GFP}^{+} \mathrm{iPSCs}$ and $\mathrm{PKH} 26^{+} \mathrm{NSCs}$ were $116 \times 10^{5}$ cells and $13 \times 10^{5}$ cells, respectively. In the presence of $\mathrm{BRQ}$, the number of $\mathrm{GFP}^{+} \mathrm{ESCs}$ and $\mathrm{GFP}^{+} \mathrm{iPSCs}$ decreased significantly $\left(5 \times 10^{5}\right.$ cells and $2 \times 10^{5}$ cells, respectively); however, $7 \times 10^{5}$ $\mathrm{PKH} 26^{+}$NSCs were detected in both cultures. These data revealed that BRQ killed 
more than $97 \%$ of the PSCs, whereas approximately $60 \%$ of the NSCs survived in the same culture (Fig. 4C, 4D).

I further examined whether BRQ selectively eliminates PSCs from co-cultures with PSC-NSCs. Three days after addition of BRQ, I immunolabeled the cells for Nanog and Nestin. In the presence of $10 \mu \mathrm{M} B R Q$, few Nanog-positive cells were detected in culture, whereas many surviving cells were positive for Nestin (Fig. 4E, 4F, Supporting information Fig. 8C, 8D), confirming the specific elimination of PSCs by BRQ.

\section{BRQ-pretreated PSCs lost tumorigenicity in vivo}

I examined the tumorigenicity of BRQ-pretreated PSCs. Two days after culturing the PSCs with DMSO alone or with $10 \mu \mathrm{M}$ BRQ, I injected the surviving cells subcutaneously into the hips of NOD/SCID mice. Four weeks after transplantation, the BRQ-pretreated cells did not grow, whereas the DMSO-treated cells formed large tumours (Fig. 5A). The tumour sizes of the DMSO- and BRQ-pretreated PSCs were $0.44 \mathrm{~cm}^{3}$ and $0.07 \mathrm{~cm}^{3}$, respectively, in the ESCs and $0.78 \mathrm{~cm}^{3}$ and $0.05 \mathrm{~cm}^{3}$, respectively, in the iPSCs (Fig. 5B). I then made sections and immunolabeled them for the PSC markers Oct4 and Nanog and for the differentiation markers for the three germ layers Nestin, $\beta$ III tubulin, ATF, and SMA. Although a large number of Oct4 ${ }^{+}$and $\mathrm{Nanog}^{+}$cells were detected in the DMSO-treated tumours, I observed no Oct4 ${ }^{+}$cells and only a small number of $\mathrm{Nanog}^{+}$cells in BRQ-treated grafts (Fig. 5C, 5D). In contrast, I found differentiated cells expressing markers for the three germ layers in every tumour (Fig. 5C, 5D). Notably, I recognized no sign of tumorigenesis in mice transplanted with 


\begin{abstract}
BRQ-pretreated PSCs, even 6 months after transplantation. Together, these data indicated that BRQ pretreatment efficiently prevented tumorigenesis in PSCs in culture.
\end{abstract}

\title{
BRQ administration prevented teratoma formation in vivo
}

Given that DHODH inhibitors have been shown to prevent tumorigenesis in many types of cancers, ${ }^{14-18}$ I examined whether BRQ prevents PSC tumorigenesis. I transplanted PSCs subcutaneously into the hips of NOD/SCID mice and allowed the tumours to reach a size greater than $100 \mathrm{~mm}^{3}$ before initiating intraperitoneal injection of BRQ once every 3 days. Three days after the fifth injection, I removed the tumours from the mice. As shown in Fig. 6A and 6B, BRQ administration strongly prevented tumour growth without any visible side effects in the mice. The sizes of the DMSO- and BRQtreated teratomas were $0.55 \mathrm{~cm}^{3}$ and $0.12 \mathrm{~cm}^{3}$, respectively, for the ESCs and $1.37 \mathrm{~cm}^{3}$ and $0.32 \mathrm{~cm}^{3}$, respectively, for the iPSCs. I then made sections and immunolabeled them for the proliferation marker Ki67, PSC markers (Oct4 and Nanog), and differentiation markers (ATF, $\beta$ III tubulin, and SMA). I confirmed that the number of $\mathrm{Ki} 7^{+}$proliferating cells was greatly decreased in the BRQ-treated tumours compared with the number in the DMSO-treated tumours (Fig. 6C; Supporting information Fig. 9). I also observed that the number of both $\mathrm{Oct}^{+}$and $\mathrm{Nanog}^{+}$cells was significantly decreased in the BRQ-treated tumours, whereas the DMSO-treated tumours contained many cells expressing these PSC markers (Fig. 6D, 6E). In contrast, both DMSO- and BRQ-treated tumours similarly contained differentiated cells expressing ATF, $\beta$ III tubulin, and SMA (Fig. 6D, 6E). Thus, these data revealed that BRQ prevented tumorigenesis by specifically eliminating undifferentiated PSCs in tumours without inducing any obvious cytotoxicity in the differentiated cells and mice. 


\section{Discussion}

Before proceeding with successful cell therapy using PSCs, it is essential to establish methods that prevent teratoma formation caused by contaminated PSCs. The results of this study demonstrated that the DHODH inhibitor BRQ specifically eliminated PSCs by inducing cell cycle arrest, cell death, and stemness loss without inducing any cytotoxicity in co-cultured NSCs. Moreover, BRQ prevented not only the tumorigenesis of the pretreated PSCs but also the growth of teratomas in vivo, although the PSCderived differentiated cells were unaffected. It is essential to evaluate whether DHODH inhibitors such as BRQ can be used as essential fundamental agents for PSC-based regenerative medicine using human PSCs, as mouse and human PSCs have been reported to be metabolically different. ${ }^{27}$

The finding that BRQ specifically eliminated PSCs without inducing any toxicity against NSCs or differentiated cells raised questions regarding the mechanism involved. I found that the dhodh/uckl expression ratio in PSCs was significantly higher than that in NSCs and differentiated cells, indicating that PSCs preferentially use the de novo pyrimidine synthesis pathway rather than the salvage pathway. This is consistent with findings that PSCs largely depend on exogenous glutamine, the substrate for the de novo pathway, to maintain their rapid proliferation. ${ }^{28,29}$ Therefore, it is of interest to investigate the molecular mechanisms that increase the $d h o d h / u c k l$ expression ratio in PSCs.

I demonstrated that BRQ induced the Crm1-dependent nuclear export of Nanog and Oct4 in PSCs. DHODH inhibitors have been shown to induce nuclear export of transcription factors, including NF- $\mathrm{BB}$ and Sox2, by preventing the O-GlcNAcyation of these factors, ${ }^{18,30}$ suggesting that the nuclear localization of both Nanog and Oct4 is 
regulated by their O-GlcNAcylation and by Crm1. Indeed, Nanog contains the nuclear export signal (NES), a target site of Crm1, and a Eukaryotic Linear Motif (http://elm.eu.org) analysis identified a potential NES in Oct4. ${ }^{31} \mathrm{O}-$ GlcNAcylation of Oct4 was demonstrated to be essential for the maintenance of ESCs. ${ }^{32}$ Although Nanog was unlikely to be O-GlcNAcylated, O-GlcNAcylated ESRRB, which regulates the self-renewal and pluripotency of PSCs, has been shown to associate strongly with Nanog and Oct4 and increase their transcriptional activity. ${ }^{33,34}$ These findings suggested that O-GlcNAcylation completely regulates the nuclear localization and activity of core PSC factors. Thus, the detailed mechanism by which O-GlcNAcylation and Crm1 regulate the nuclear localization of PSC factors is an interesting subject for future investigations.

\section{Conclusion}

The results of this study demonstrated that BRQ selectively induced cell cycle arrest and cell death in PSCs but was less toxic to non-PSCs. It was also shown that BRQpretreated PSCs did not form teratomas, while BRQ administration prevented teratoma growth with the eradication of PSC marker-positive cells in tumours. Thus, these findings revealed that DHODH inhibitors such as BRQ can be used as fundamental chemicals for PSC-based cell therapy.

\section{Acknowledgement}

The author thanks Shinji Masui for E14tg2a and a general method for PSC culture, and Hisataka Sabe for C2C12. 


\section{Disclosure of potential conflicts of interest}

The author indicated no potential conflicts of interests.

\section{References}

1. Yamanaka S, Blau HM. Nuclear reprogramming to a pluripotent state by three approaches. Nature 2010;465:704-712.

2. Gokhale PJ, Andrews PW. The development of pluripotent stem cells. Curr Opin Genet Dev 2012;22:403-408.

3. Cahan P, Daley GQ. Origins and implications of pluripotent stem cell variability and heterogeneity. Nat Rev Mol Cell Biol 2013;14:357-368.

4. Avior Y, Sagi I, Benvenisty N. Pluripotent stem cells in disease modelling and drug discovery. Nat Rev Mol Cell Biol 2016;17:170-182.

5. Ilic D, Ogilvie C. Concise Review: Human Embryonic Stem Cells-What Have We Done? What Are We Doing? Where Are We Going? Stem Cells 2017;35:17-25.

6. Tang C, Lee AS, Volkmer JP, et al. An antibody against SSEA-5 glycan on human pluripotent stem cells enables removal of teratoma-forming cells. Nat Biotechnol 2011;29:829-834.

7. Shiraki N, Shiraki Y, Tsuyama T, et al. Methionine metabolism regulates maintenance and differentiation of human pluripotent stem cells. Cell Metab 2014;19:780-794.

8. Kuang Y, Miki K, Parr CJC, et al. Efficient, Selective Removal of Human Pluripotent Stem Cells via Ecto-Alkaline Phosphatase-Mediated Aggregation of Synthetic Peptides. Cell Chem Biol 2017;24:685-694.

9. Ben-David U, Gan QF, Golan-Lev T, et al. Selective elimination of human pluripotent stem cells by an oleate synthesis inhibitor discovered in a high-throughput screen. Cell 
Stem Cell 2013;12:167-179.

10. Cliff TS, Dalton S. Metabolic switching and cell fate decisions: implications for pluripotency, reprogramming and development. Curr Opin Genet Dev 2017;46:44-49.

11. Tsogtbaatar E, Landin C, Minter-Dykhouse K, et al. C.D.L. Energy Metabolism Regulates Stem Cell Pluripotency. Front Cell Dev Biol 2020;8:87.

12. Tohyama S, Fujita J, Hishiki T, et al. Glutamine Oxidation Is Indispensable for Survival of Human Pluripotent Stem Cells. Cell Metab 2016;23:663-674.

13. Scalise M, Pochini L, Galluccio M, et al. Glutamine Transport and Mitochondrial Metabolism in Cancer Cell Growth Front Oncol 2017;7:306.

14. White RM, Cech J, Ratanasirintrawoot S, et al. DHODH modulates transcriptional elongation in the neural crest and melanoma. Nature 2011;471:518-522.

15. Sykes DB, Kfoury YS, Mercier FE, et al. Inhibition of Dihydroorotate Dehydrogenase Overcomes Differentiation Blockade in Acute Myeloid Leukemia. Cell 2016;167:171186.

16. Brown KK, Spinelli JB, Asara JM, et al. Adaptive Reprogramming of De Novo Pyrimidine Synthesis Is a Metabolic Vulnerability in Triple-Negative Breast Cancer. Cancer Discov 2017;7:391-399.

17. Koundinya M, Sudhalter J, Courjaud A, et al. Dependence on the Pyrimidine Biosynthetic Enzyme DHODH Is a Synthetic Lethal Vulnerability in Mutant KRASDriven Cancers. Cell Chem Biol 2018;25:705-717.

18. Echizenya S, Ishii Y, Kitazawa S, et al. Discovery of a new pyrimidine synthesis inhibitor eradicating glioblastoma-initiating cells. Neuro-Oncol 2020;22:229-239.

19. Johe KK, Hazel TG, Muller T, et al. Single factors direct the differentiation of stem 
cells from the fetal and adult central nervous system. Genes Dev 1996;10:3129-3140.

20. Hide T, Takezaki T, Nakatani Y, et al. Combination of a ptgs2 inhibitor and an epidermal growth factor receptor-signaling inhibitor prevents tumorigenesis of oligodendrocyte lineage-derived glioma-initiating cells. Stem Cells 2011;29:590-599.

21. Kodama HA, Amaga Y, Koyama H, et al. Hormonal responsiveness of a preadipose cell line derived from newborn mouse calvaria. J Cell Physiol 1982;112:83-88.

22. Kondo T, Raff M. Chromatin remodeling and histone modification in the conversion of oligodendrocyte precursors to neural stem cells. Genes Dev 2004;18:2963-2972.

23. Tsukamoto Y, Ohtsu N, Echizenya S, et al. Chemical Screening Identifies EUrd as a Novel Inhibitor Against Temozolomide-Resistant Glioblastoma-Initiating Cells. Stem Cells 2016;34:2016-2025.

24. Kondo T, Raff M. The Id4 HLH protein and the timing of oligodendrocyte differentiation. EMBO J 2000;19:1998-2007.

25. Takanaga H, Tsuchida-Straeten N, Nishide K, et al. Gli2 Is A Novel Regulator of Sox2 Expression In Telencephalic Neuroepithelial Cells. Stem Cells 2009;27:165-174.

26. Balestri F, Barsotti C, Lutzemberger L, et al. Key role of uridine kinase and uridine phosphorylase in the homeostatic regulation of purine and pyrimidine salvage in brain. Neurochem Int 2007;51:517-523.

27. Zhou W, Choi M, Margineautu D, et al. HIF1 $\alpha$ induced switch from bivalent to exclusively glycolytic metabolism during ESC-to-EpiSC/hESC transition. EMBO J 2012;31:2103-2116.

28. Ryu JM, Lee SH, Seong JK, et al. Glutamine contributes to maintenance of mouse embryonic stem cell self-renewal through PKC-dependent downregulation of HDAC1 and DNMT1/3a. Cell Cycle. 2015;14:3292-3305. 
29. Lees JG, Gardner DK, Harvey AJ. Nicotinamide adenine dinucleotide induces a bivalent metabolism and maintains pluripotency in human embryonic stem cells. Stem Cells. 2020;38:624-638.

30. Yang WH, Park SY, Nam HW, et al. NFkappaB activation is associated with its OGlcNAcylation state under hyperglycemic conditions. Proc Natl Acad Sci USA 2008;105:17345-17350.

31. Park SW, Do HJ, Huh SH, et al. Identification of a putative nuclear export signal motif in human NANOG homeobox domain. Biochem Biophys Res Commun 2012;421:484-489.

32. Jang H, Kim TW, Yoon S, et al. O-GlcNAc regulates pluripotency and reprogramming by directly acting on core components of the pluripotency network. Cell Stem Cell 2012;11:62-74.

33. Speakman CM, Domke TC, Wongpaiboonwattana W, et al. Elevated O-GlcNAc levels activate epigenetically repressed genes and delay mouse ESC differentiation without affecting naïve to primed cell transition. Stem Cells 2014;32:2605-2615.

34. Hao Y, Fan X, Shi Y, et al. Next-generation unnatural monosaccharides reveal that ESRRB O-GlcNAcylation regulates pluripotency of mouse embryonic stem cells. Nat Commun 2019;10:4065.

\section{Figure legends}

\section{Figure 1. BRQ specifically eliminates PSCs}

(A): Dose-dependent effects of the DHODH inhibitors teriflunomide (diamond), leflunomide (square), vidofludimus (cross), and BRQ (circle) against ESCs (left panel) and iPSCs (right panel). (B): Dose-dependent effects of BRQ against NSCs (closed circle), astrocytes (closed triangle), and ESCs (open circle). (C): Dose-dependent 
effects of BRQ against PA6 (closed circle), C2C12 (closed triangle), and ESCs (open circle). (D): Rescue effects of ribonucleosides, uridine (circles), adenosine (diamond), guanosine (square), and cytidine (triangle) against BRQ $(10 \mu \mathrm{M})$-dependent cytotoxicity towards ESCs (left panel) and iPSCs (right panel). (E): Rescue effects of nucleotide diphosphates, UDP (circles), CDP (diamond), ADP (square), and GDP (triangle) against BRQ $(10 \mu \mathrm{M})$-dependent cytotoxicity towards ESCs (left panel) and iPSCs (right panel). All experiments were repeated at least three times with similar results. The error bars indicate $\pm \mathrm{SD}$. A $t$ test was used for statistical significance. $* * P$ $<.01, * * * P<.001$.

\section{Figure 2. BRQ induced cell cycle arrest and cell death in PSCs}

(A): Proportion of BrdU+ cells among PSCs, ESCs, and iPSCs cultured with DMSO or BRQ $(10 \mu \mathrm{M})$ for 2 days. (B): Proportion of CASP3+ cells among PSCs, ESCs, and iPSCs cultured with DMSO or BRQ $(10 \mu \mathrm{M})$ for 2 days. (C): Proportion of annexin V+ cells among PSCs, ESCs, and iPSCs cultured with DMSO or BRQ $(10 \mu \mathrm{M})$ for 2 days. (D): Quantitative RT-PCR analysis of the expression of sox2, oct4 and nanog in PSCs, ESCs, and iPSCs cultured with DMSO or BRQ $(10 \mu \mathrm{M})$ for 2 days. The mRNA levels are shown as fold changes over mRNA levels in the presence of DMSO. All experiments were repeated at least three times with similar results. Error bar: \pm SD. Statistical significance was determined by the $t$ test. ${ }^{*} P<.05, * * P<.01, * * * P<.001$.

\section{Figure 3: DHODH knockdown induced cell cycle arrest and cell death in PSCs}

(A): Proportion of Ki67+ cells among either control sh- or dhodh sh-expressing ESCs (left panel) and iPSCs (right panel). (B): Proportion of Casp3+ cells among either control 
sh- or dhodh sh-expressing ESCs (left panel) and iPSCs (right panel). All experiments were repeated at least three times with similar results. Error bar: \pm SD. Statistical significance was determined by the $t$ test. ${ }^{*} P<.05,{ }^{*} * P<.01, * * * P<.001$.

\section{Figure 4. BRQ selectively eliminated PSCs co-cultured with NSCs}

(A, B): Representative immunostained images of co-cultured PSCs, ESCs (A) and iPSCs (B) with NSCs in the presence of DMSO or BRQ $(1$ and $10 \mu \mathrm{M})$ for Nestin and Nanog. Scale bar: $100 \mu \mathrm{m}$. (C, D): BRQ cytotoxicity against ESCs (C), iPSCs (D), and NSCs. $(\mathbf{E}, \mathbf{F})$ : BRQ cytotoxicity against ESCs (E), iPSCs (F), and PSC-NSCs. Error bar: \pm SD. Statistical significance was determined by the $t$ test. $* P<.05, * * P<.01, * * * P<.001$.

\section{Figure 5. BRQ-pretreated PSCs lost tumorigenicity in vivo}

(A): Representative photographs of teratomas formed by ESCs and iPSCs, which were pretreated with DMSO alone or BRQ $(10 \mu \mathrm{M})$. Scale bar: $1 \mathrm{~cm}$. (B): Size of the teratoma formed by the pretreated ESCs and iPSCs. Statistical significance was determined by the $t$ test. Error bar: \pm SD. $* * * P<.001$. (C, D): Representative immunostaining images of teratomas formed by pretreated ESCs (C) and iPSCs (D) for Oct4 (red), Nanog (green), AFP (green), $\beta$ III tubulin (red), and SMA (green). Nuclei were counterstained with DAPI (blue). Scale bar: $100 \mu \mathrm{m}$.

\section{Figure 6. BRQ prevented tumour growth of the transplanted PSCs}

(A): Representative photographs of teratomas formed by ESCs (left panels) and iPSCs (right panels) in mice treated with DMSO alone or BRQ (25 mg/kg). (B): Size of teratomas formed by ESCs and iPSCs in mice treated with DMSO or BRQ. (C): 
Proportion of Ki67+ cells in teratomas formed by ESCs and iPSCs in mice treated with DMSO or BRQ. (D, E): Representative immunostaining images of teratomas formed by ESCs (D) and iPSCs (E) in mice treated with DMSO or BRQ for Oct4 (red), Nanog (green), AFP (green), $\beta$ III tubulin (red), and SMA (green). Nuclei were counterstained with DAPI (blue). Scale bar: $100 \mu \mathrm{m}$. 
A
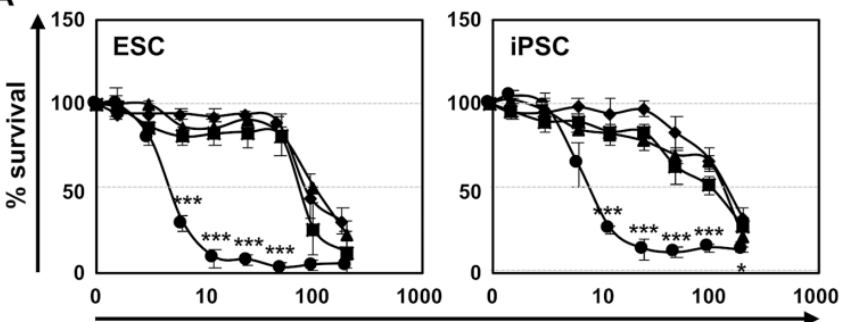

DHODH inhibitor $(\mu \mathrm{M})$

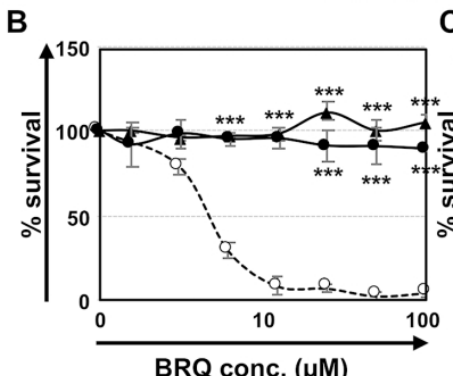

C

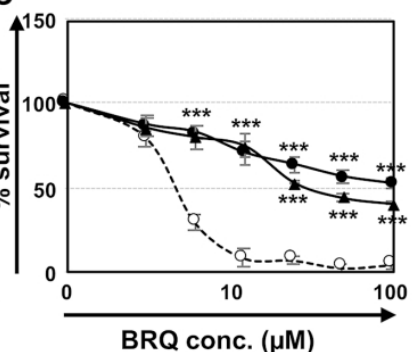

D
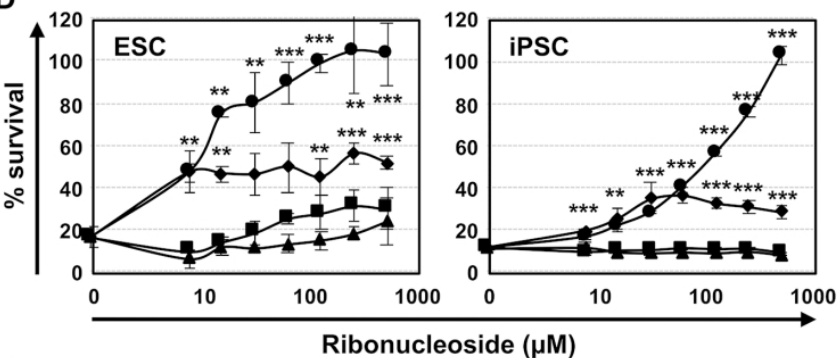

E
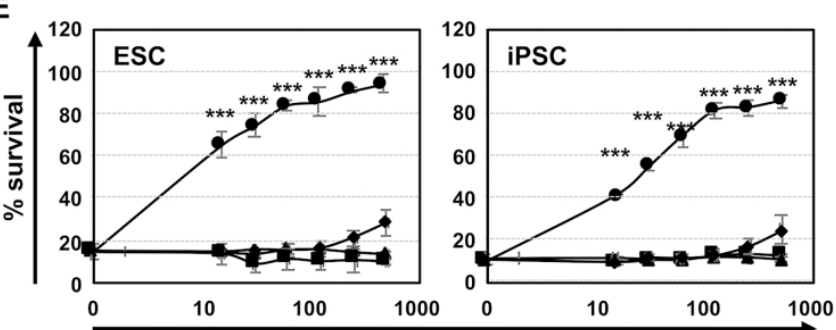

Nucleotide diphosphate $(\mu \mathrm{M})$

Figure 1. BRQ specifically eliminates PSCs 
A

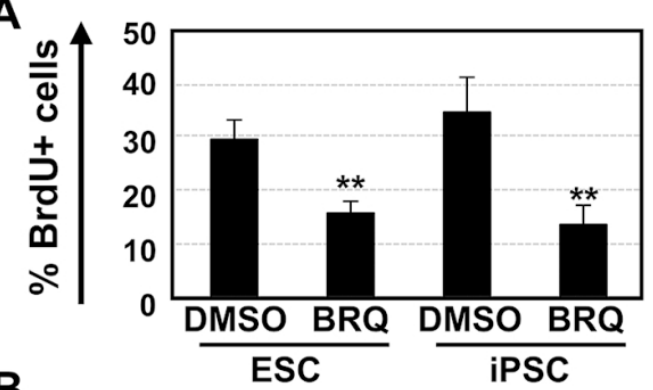

B

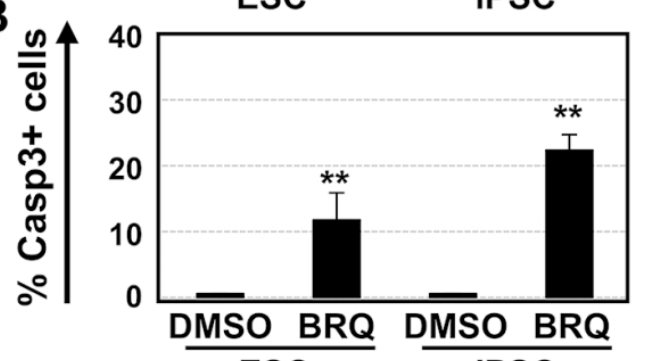

C

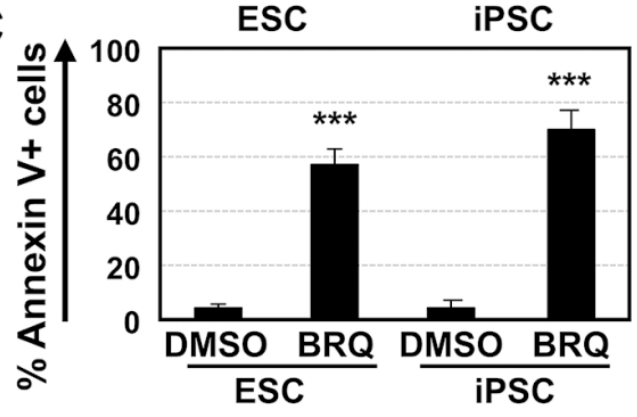

D
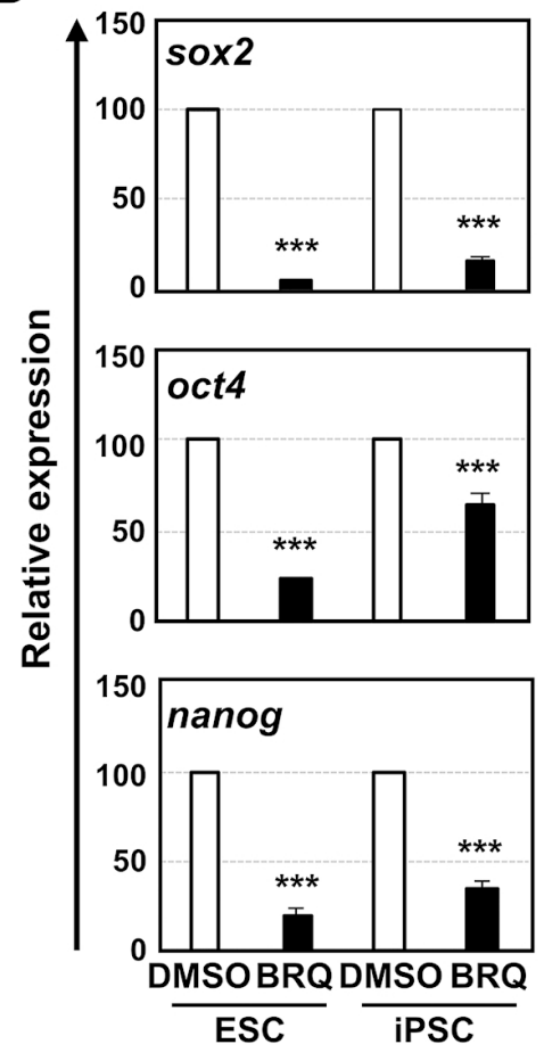

Figure 2. BRQ induced cell cycle arrest and cell death in PSCs

$118 \times 108 \mathrm{~mm}(300 \times 300 \mathrm{DPI})$ 


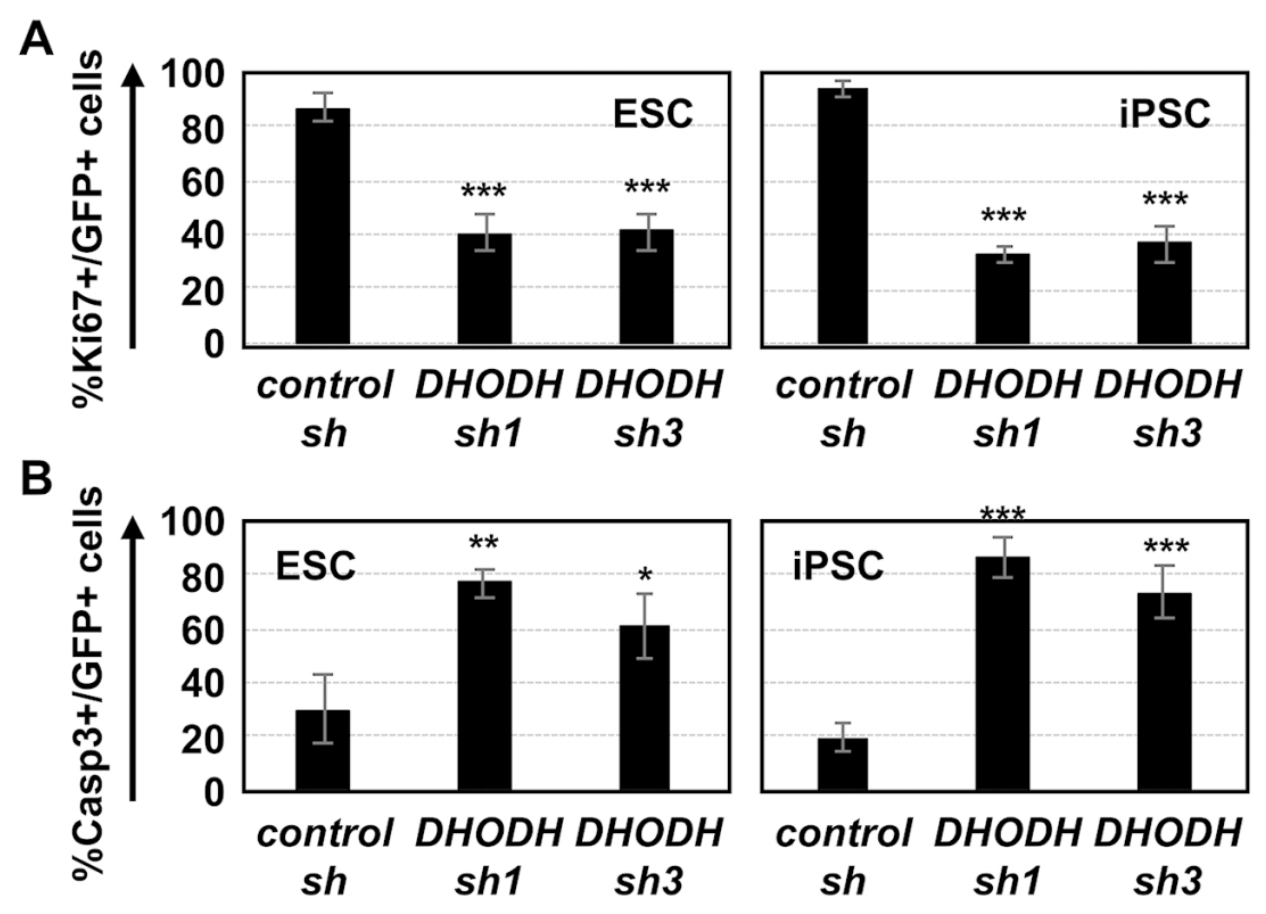

Figure 3: DHODH knockdown induced cell cycle arrest and cell death in PSCs $113 \times 82 \mathrm{~mm}(300 \times 300$ DPI) 


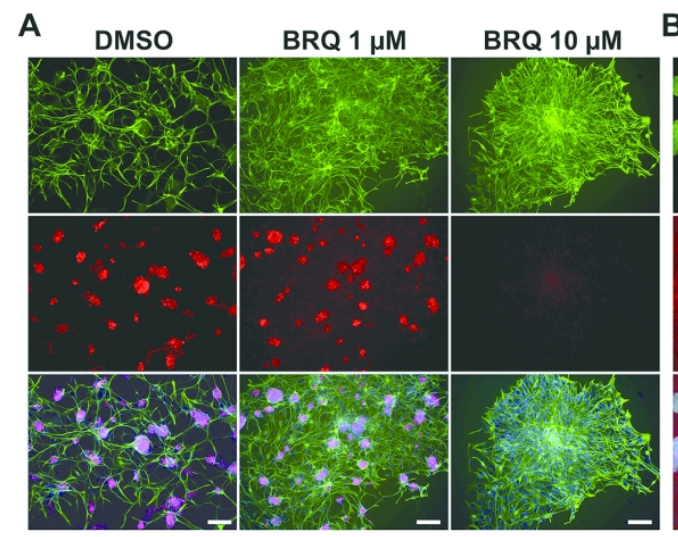

C

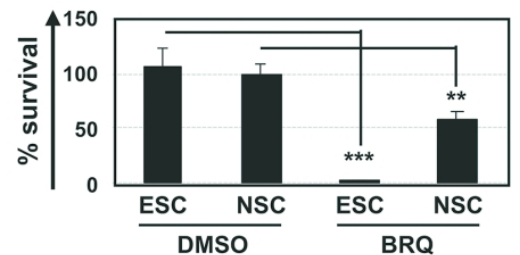

E

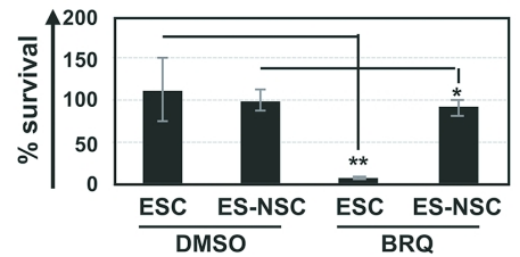

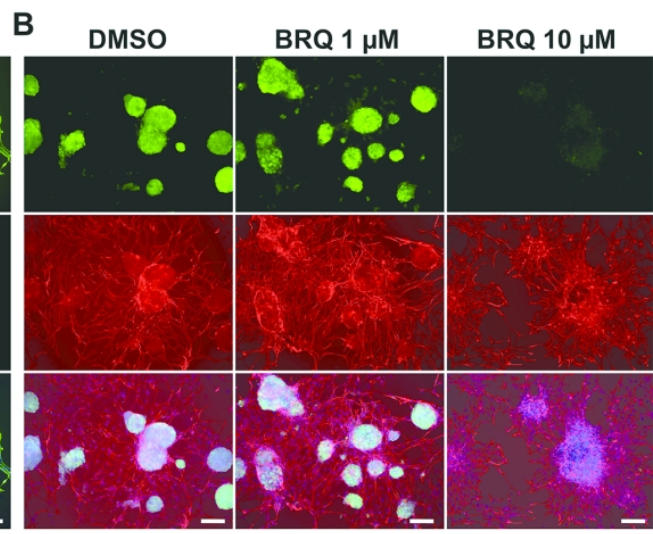

Nestin/Nanog/Nuclei

D

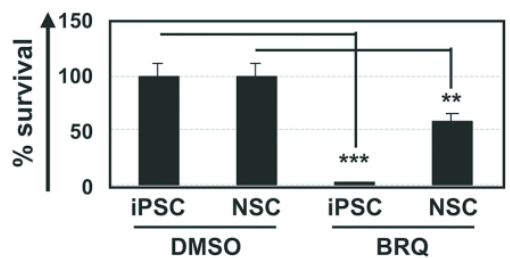

$\mathbf{F}$

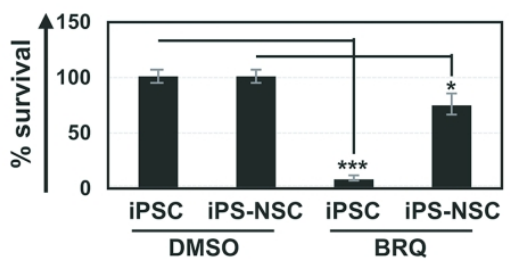

Figure 4. BRQ selectively eliminated mPSCs co-cultured with NSCs $173 \times 157 \mathrm{~mm}(300 \times 300$ DPI $)$ 


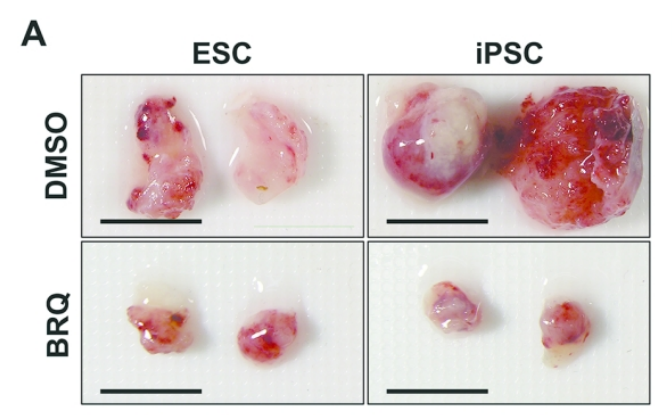

B

\section{C}
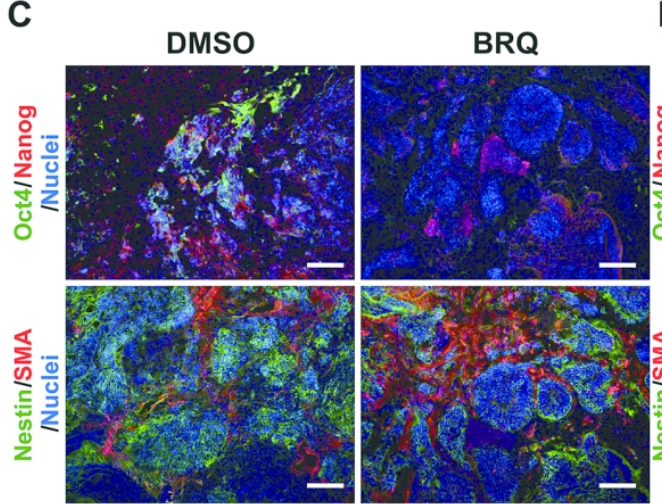

D
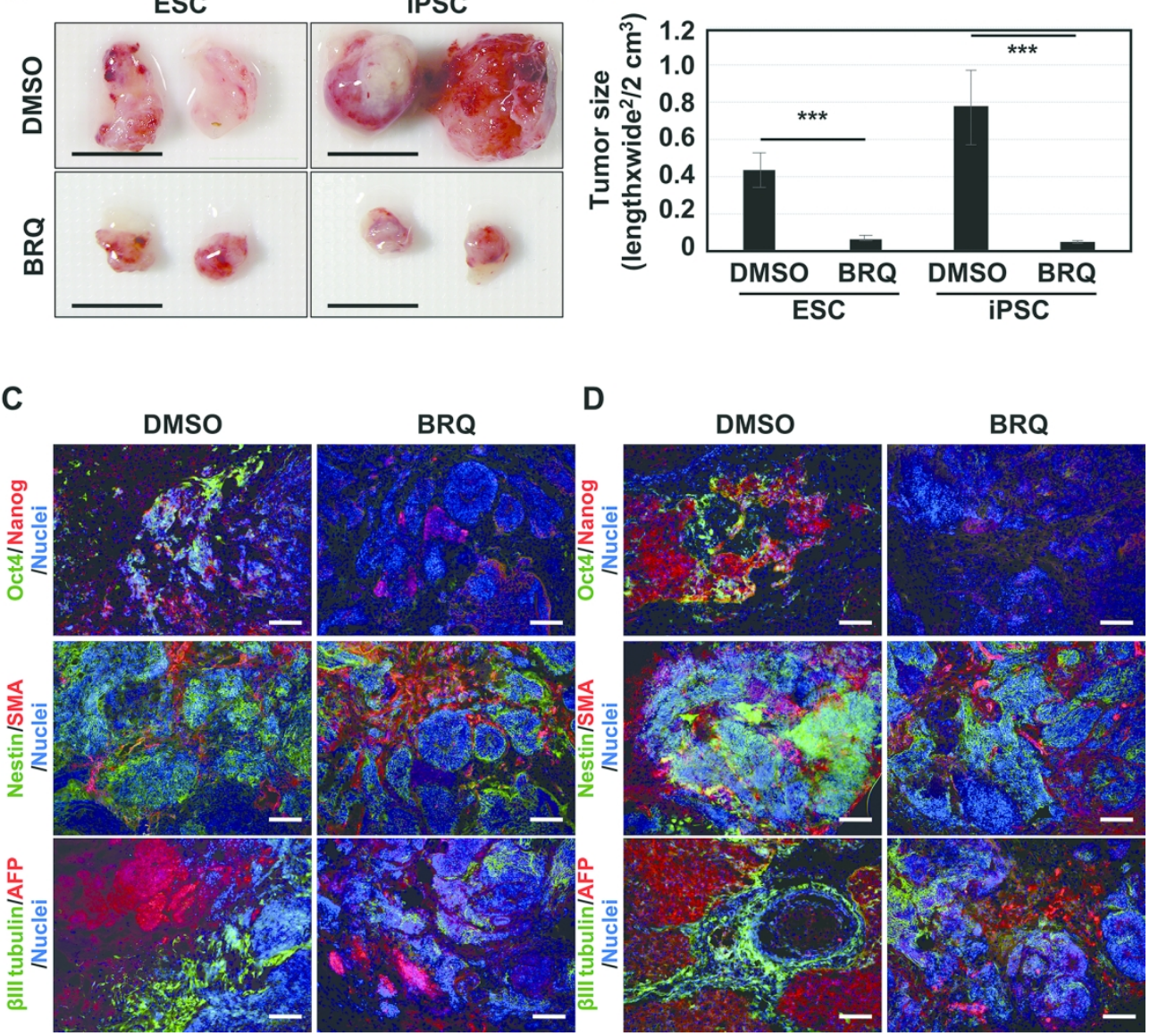

Figure 5. BRQ-pretreated mPSCs lost tumorigenicity in vivo $154 \times 142 \mathrm{~mm}(300 \times 300 \mathrm{DPI})$ 
A
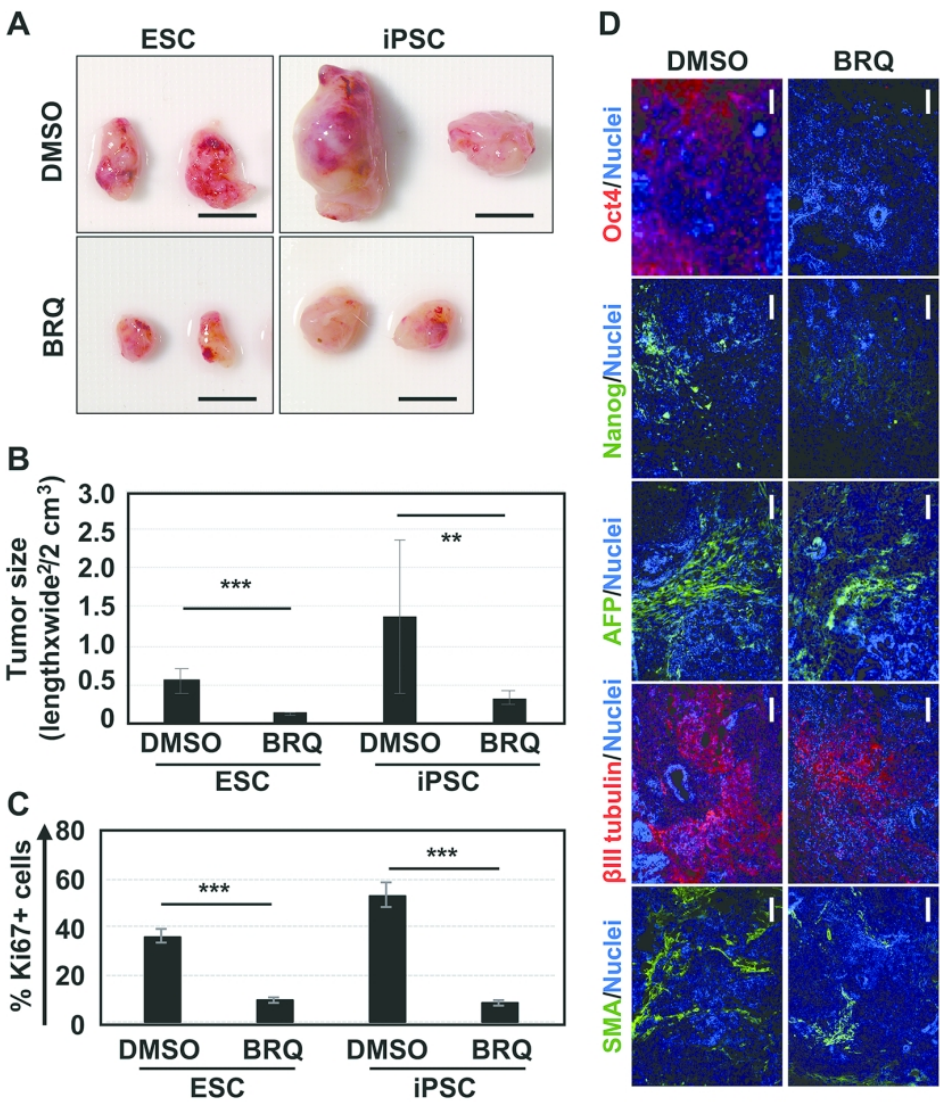

E DMSO BRQ

Figure 6. BRQ prevented tumour growth of the transplanted mPSCs $167 \times 145 \mathrm{~mm}(300 \times 300 \mathrm{DPI})$ 


\section{Supplementary Information}

\section{Supplementary Methods}

\section{Western blotting}

Western blotting was performed as previously described ${ }^{23}$. In brief, the FLAG-tagged mouse DHODH expression vector was transfected with a control sh (cont) or $d h o d h$ sh1-3 expression vector into Cos7 cells. Cell extracts were harvested 2 days after transfection and then analysed by Western blotting using anti-FLAG M2 (10 $\mu \mathrm{g} / \mathrm{ml}$, Sigma-Aldrich, St. Louis, MO, www.sigmaaldrich.com) and anti-GAPDH (loading control, 1:5000, Proteintech, Rosemont, IL) antibodies. Antibodies were detected with horseradish peroxidase-conjugated anti-mouse IgG (1:5000, Santa Cruz Biotechnology Inc., Santa Cruz, CA, http://www.scbt.com).

\section{Construction of knockdown vectors for $u c k 1$ and $u c k 2$}

To knock down mouse $u c k 1$ and $u c k 2$, two short hairpin (sh) sequences of each were generated using InvivoGen's siRNA Wizard (http://www.sirnawizard.com/). These sh sequences were inserted into a psiRNA-DUO expression vector (InvivoGen, San Diego, CA, www.invivogen.com) to produce psiRNA-DUO-muck1 sh1, -muck1 sh2, -muck2 sh1, -muck2 sh2, and -muck1 sh1 and -muck2 sh1. The knockdown efficiency of these vectors was analysed by immunostaining (Supporting information Fig. 6A, 6B). The target sequences for mouse $u c k 1$ and $u c k 2$ were as follows: $u c k 1 \operatorname{sh} 1$ and $\operatorname{sh} 2$ were 5 'GAGCCAGGACTGCTTCTACAA-3' and 5'-GGACTGCTTCTACAAGGTTCT-3', respectively, and $u c k 2 s h 1$ and sh2 were 5'-GCCGATGTGATCATTCCTAGA-3' and 5'-GCAGACGAACGGCTATCTCAA-3', respectively. 
The nucleotide sequences were verified using the BigDye Terminator Kit version 3.1 (Thermo Fisher Scientific, Grand Island, New York) and an ABI sequencer model 3130xl (Thermo Fisher Scientific, Grand Island, New York). Cells were transfected with the vectors using Lipofectamine 3000 (Thermo Fisher Scientific, Grand Island, New York) according to the manufacturer's protocol.

To examine the knockdown efficiency of shRNAs, the transfected cells were immunolabeled with rat monoclonal anti-GFP (1:500, Nacalai Tesque) and rabbit polyclonal anti-DHODH (1:100, Proteintech, Rosemont, IL), anti-Uck1 (1:100, Proteintech, Rosemont, IL) or anti-Uck2 (1:100, Proteintech, Rosemont, IL) (Supplementary information Fig. 3B, 3C, 6A, 6B).

The $u c k 1$ and $u c k 2$ double-knockdown PSCs were established by culturing the transfected cells in the presence of zeocin $(0.1 \mathrm{mg} / \mathrm{ml})$ for one week (Supplementary information Fig. 6E, 6F).

\section{Supplementary Figure legends}

\section{Figure 1. Cytotoxicity of DHODH inhibitors against PA6 and C2C12 cells}

Dose-dependent effects of the DHODH inhibitors teriflunomide (diamond), leflunomide (square), vidofludimus (triangle) and BRQ (circle) against PA6 (upper panel) and C2C12 (lower panel) cells.

\section{Figure 2. BRQ increased Casp3 activation in PSCs and decreased PSC marker- positive cells}

(A): PSCs were cultured in the presence of DMSO alone or BRQ $(10 \mu \mathrm{M})$ for 2 days and then immunostained for cleaved Casp3 (red). (B): PSCs were treated as in (A) and then 
immunostained for the PSC markers Sox2, Nanog and Oct4 (green). Arrows indicate PSC marker-negative cells. Nuclei were counterstained with DAPI (blue). Scale bar: $100 \mu \mathrm{m}$.

\section{Figure 3. Knockdown efficiency of $d h o d h$ sh expression vectors}

(A): The FLAG-tagged mouse DHODH expression vector was transfected with a control sh (cont) or dhodh sh1-3 expression vector into Cos7 cells. Cell extracts were harvested 2 days after transfection and then analysed by Western blotting using anti-FLAG and antiGAPDH (loading control) antibodies. (B, C): Representative photographs of either control sh-, dhodh sh1- or dhodh sh3-expressing ESCs (B) and iPSCs (C) that were immunostained for GFP (green) and DHODH (purple). Arrows indicate GFP+/DHODHcells. All nuclei were counterstained with DAPI (blue). Scale bar, $100 \mu \mathrm{m}$.

Figure 4. Knockdown of $d h o d h$ decreased $\mathrm{Ki}^{+} 7^{+}$cells and increased $\mathrm{Casp}^{+}$cells in PSCs

(A): Representative photographs of either control sh-or $d h o d h$ sh-expressing PSCs that were immunostained for GFP (green) and Ki67 (red). (B): Representative photographs of either control sh- or dhodh sh-expressing PSCs that were immunostained for GFP (green) and cleaved Casp3 (red). All nuclei were counterstained with DAPI (blue). Scale bar, $100 \mu \mathrm{m}$.

\section{Figure 5. Increased $d h o d h / u c k 1$ expression ratio in PSCs}

(A): Pyrimidine biosynthesis pathways. (B): Quantitative RT-PCR analysis of the expression of dhodh, uckl and uck2 in NSCs, ESCs, iPSCs, PA6 cells and C2C12 cells. The mRNA levels are shown as fold changes over mRNA levels in NSCs. (C): Ratio of 
dhodh/uckl in NSCs, ESCs, iPSCs, PA6 cells and C2C12 cells. (D): Ratio of DHODH/UCK1 in normal human tissues. (E): Ratio of DHODH/UCK1 in human cell lines. The black dotted line indicates that a ratio of 1 .

Figure 6. Effects of $u c k 1$ and $u c k 2$ knockdown in PSCs

(A, B): Representative photographs of either control sh-, uckl sh-or uck2 sh-expressing ESCs that were immunostained for GFP (green) and either Uck1 (purple) (A) or Uck2 (purple) (B). Arrows indicate $\mathrm{GFP}^{+} / \mathrm{Uck}^{-}$cells. $* \mathrm{GFP}^{+} / \mathrm{Uck}^{+}$cells. (C): Representative photographs of control sh-, uck1 sh1 or uck2 sh1-expressing PSCs, ESCs (left panels) and iPSCs (right panels) that were immunostained for GFP (green) and Ki67 (red). Arrows indicate $\mathrm{GFP}^{+} / \mathrm{Ki}^{+} 7^{+}$cells. (D): Representative photographs of control sh- or uck1 sh1 and uck2 sh1-expressing PSCs, ESCs and iPSCs that were immunostained for GFP (green) and Uck1 (red) (left panels) or GFP (green) and Uck2 (red) (right panels). All nuclei were counterstained with DAPI (blue). Scale bar, $100 \mu \mathrm{m}$.

\section{Figure 7. LMB prevented the BRQ-dependent nuclear export of PSC markers}

(A, B): Addition of ZVAD did not prevent BRQ-dependent nuclear export of Oct4 (green) and Nanog (red) in ESCs (A) and iPSCs (B). (C, D): Addition of LMB retained Oct4 (green) and Nanog (red) in the nucleus of BRQ-treated ESCs (C) and iPSCs (D). Nuclear PSC marker ${ }^{+}$cells are shown as a percentage of the total cells. Nuclei were counterstained with DAPI (blue). Scale bar, $50 \mu \mathrm{m}$ in A and $100 \mu \mathrm{m}$ in B.

Figure 8. PSC-derived NSC differentiation 
(A, B): PSC-derived NSCs, ES-derived NSCs (A) and iPS-derived NSCs (B) were positive for Nestin (green) and Sox2 (red). When the cells were cultured in either mitogen-free NSC medium or DMEM $+1 \%$ FCS for 1 week, they differentiated into $\beta I I I$ tubulin ${ }^{+}$neurons (green) and $\mathrm{GFAP}^{+}$astrocytes (red) in mitogen-free NSC medium (no mitogen, approximately $29 \%$ and $16 \%$, respectively) and in DMEM $+1 \%$ FCS (approximately $9 \%$ and 57\%, respectively). Arrows indicate $\beta I I I$ tubulin ${ }^{+} / \mathrm{GFAP}^{-}$cells. (C, D): Representative immunostained images of co-cultured PSCs, ESCs (C) and iPSCs (D) with PSC-NSCs in the presence of DMSO or BRQ (1 and $10 \mu \mathrm{M})$ for Nestin (green) and Nanog (red). Scale bar, $100 \mu \mathrm{m}$.

\section{Figure 9. BRQ administration decreased $\mathrm{Ki}^{+} 7^{+}$cells in teratomas}

Representative immunostaining images of teratomas that were formed by ESCs and iPSCs in mice treated with DMSO or BRQ for Ki67 (red). All nuclei were counterstained with DAPI (blue). Scale bar, $100 \mu \mathrm{m}$.

\section{Figure legend for Graphical Abstract}

This study demonstrated that Brequinar (BRQ), an inhibitor for the dihydroorotate dehydrogenase (DHODH), not only induced cell cycle arrest and cell death in PSCs in vitro but also prevented tumorigenesis by selectively eliminating PSCs in vivo. Given that BRQ was less toxic for non-PSCs, these findings identified DHODH inhibitors, such as BRQ, can be used as fundamental chemicals for PSC-based cell therapy. 


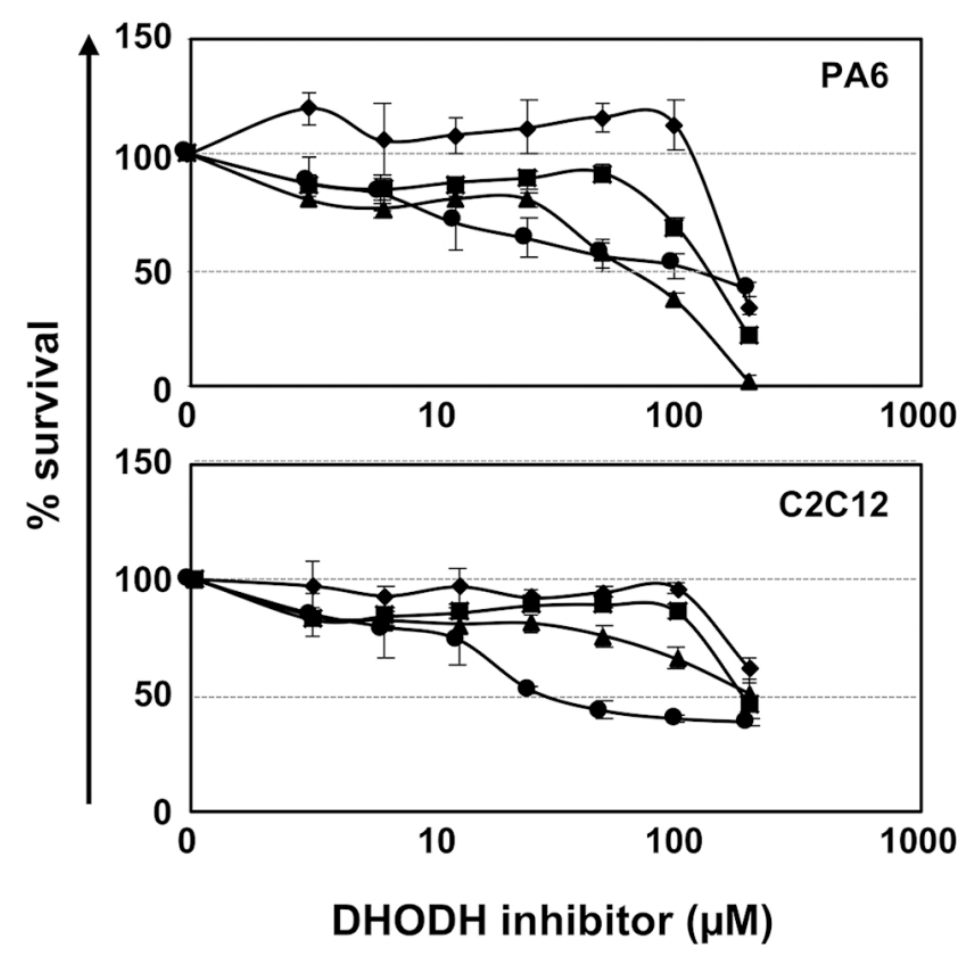

\section{- BRQ $\Delta$ Vidofludimus - Leflunomide $\bullet$ Teriflunomide}

Supplementary Figure 1. Cytotoxicity of DHODH inhibitors against PA6 and C2C12 cells $128 \times 110 \mathrm{~mm}(270 \times 270 \mathrm{DPI})$ 
A

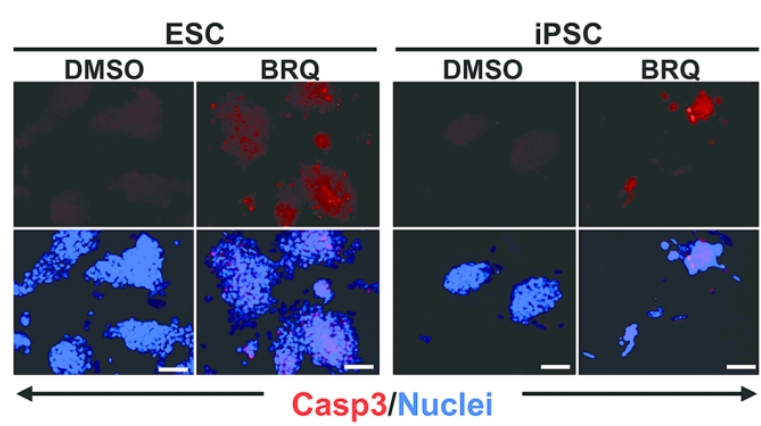

B
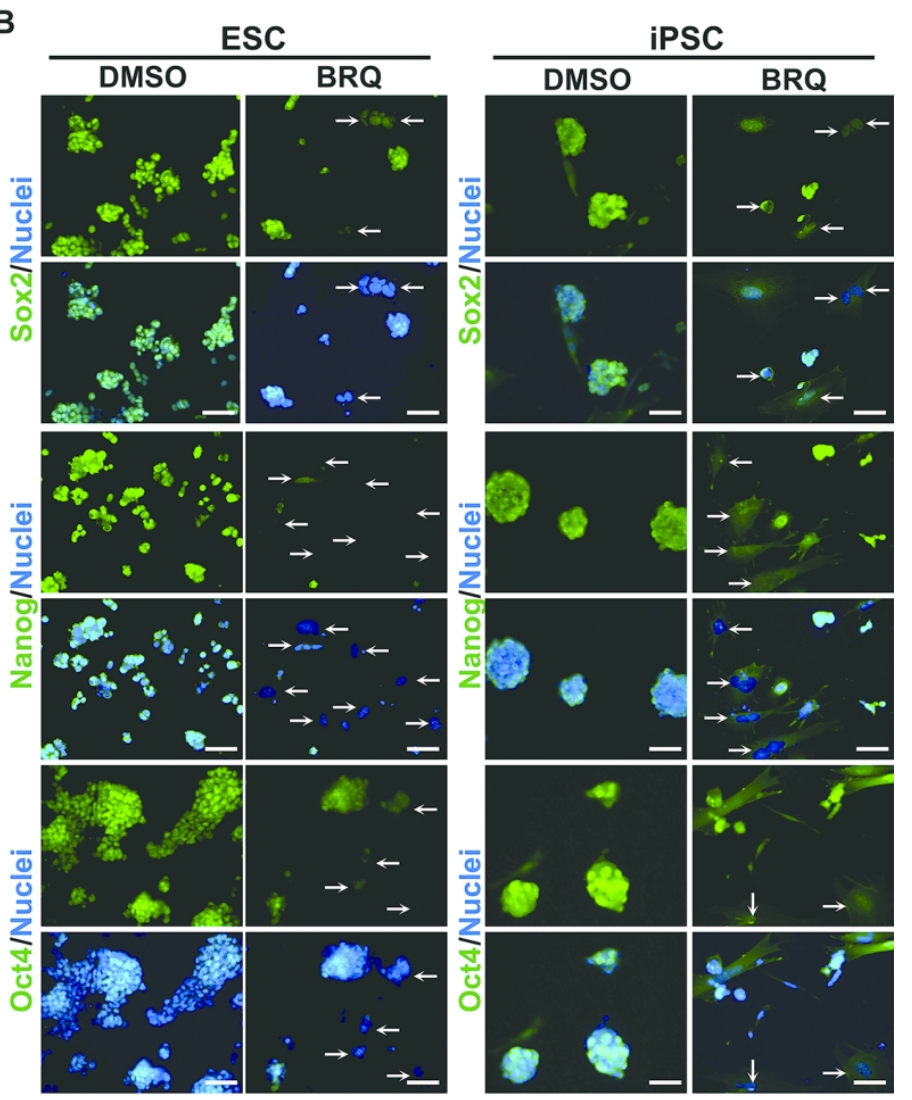

Supplementary Figure 2. BRQ increased Casp3 activation in PSCs and decreased PSC markerpositive cells $120 \times 204 \mathrm{~mm}(270 \times 270 \mathrm{DPI})$ 
A

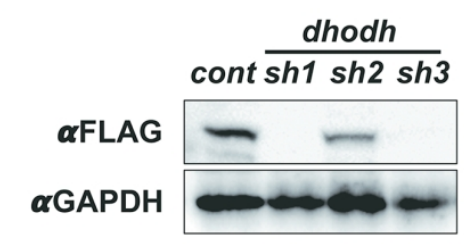

B

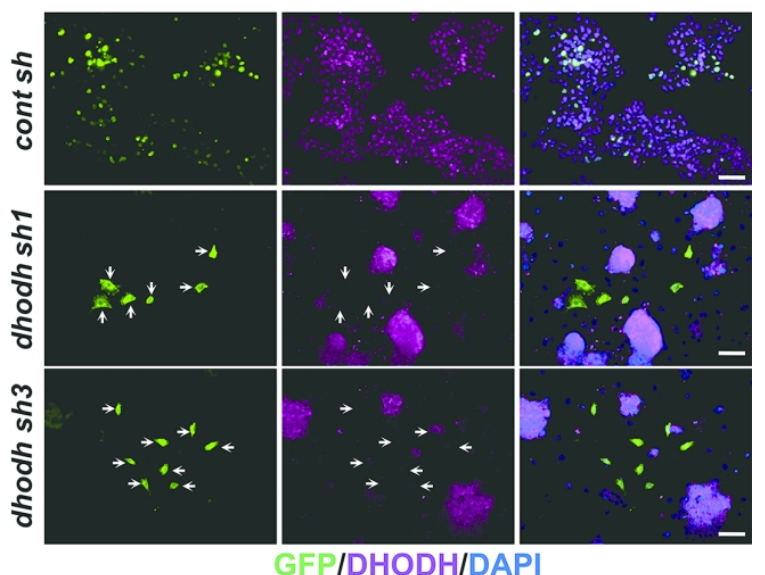

C

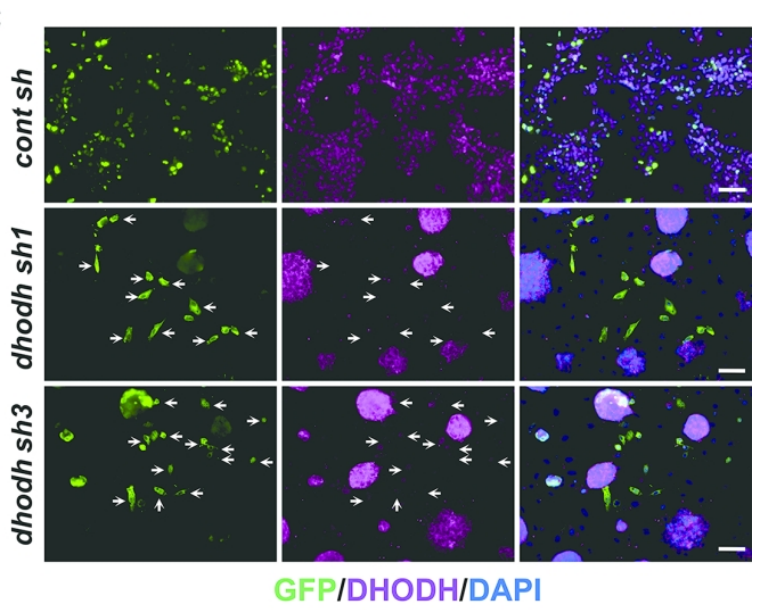

Supplementary Figure 3. Knockdown efficiency of dhodh sh expression vectors $108 \times 213 \mathrm{~mm}(270 \times 270 \mathrm{DPI})$ 
A

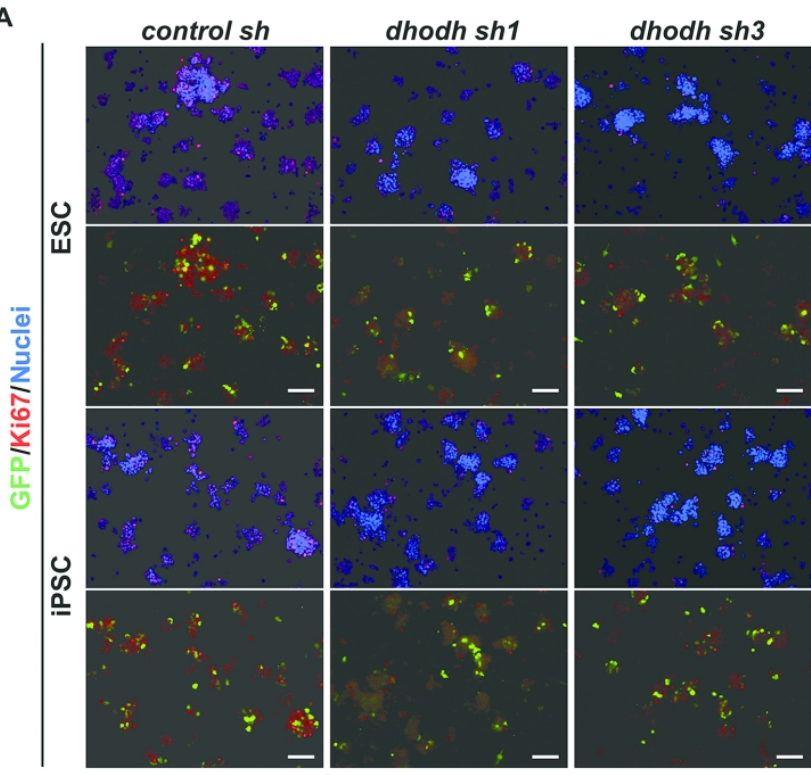

B

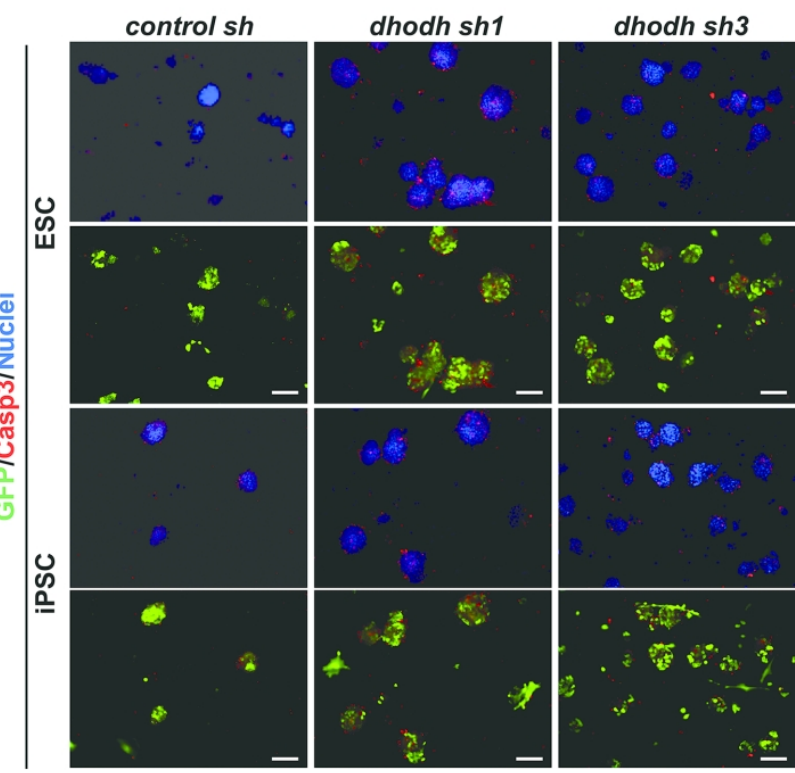

Supplementary Figure 4. Knockdown of dhodh decreased Ki67+ cells and increased Casp3 + cells in PSCs $132 \times 248 \mathrm{~mm}(270 \times 270 \mathrm{DPI})$ 
A

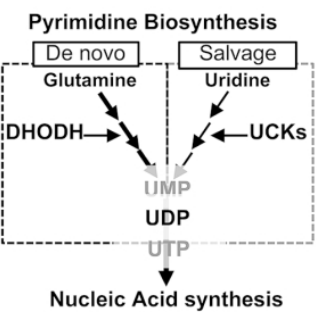

C

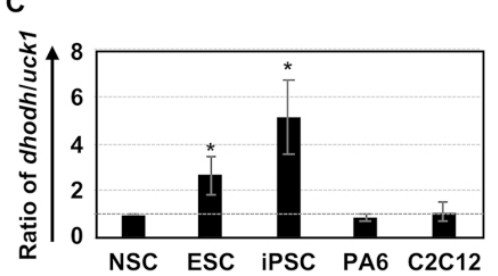

D

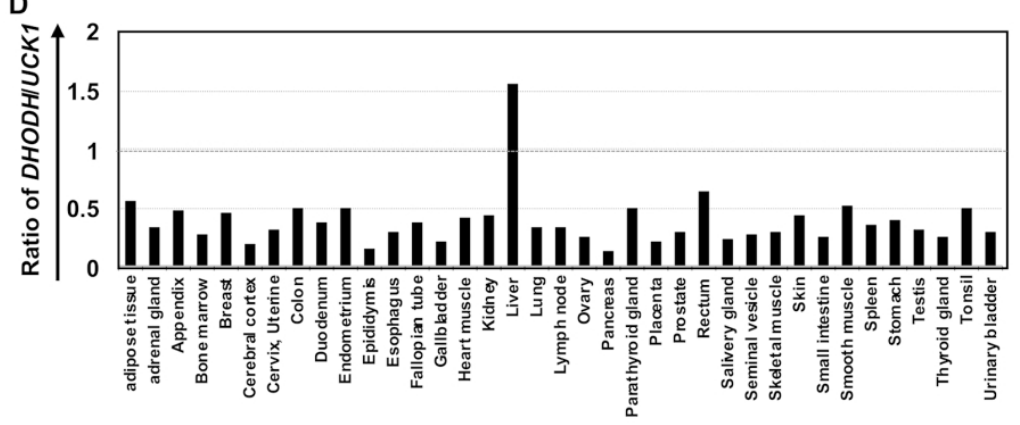

B

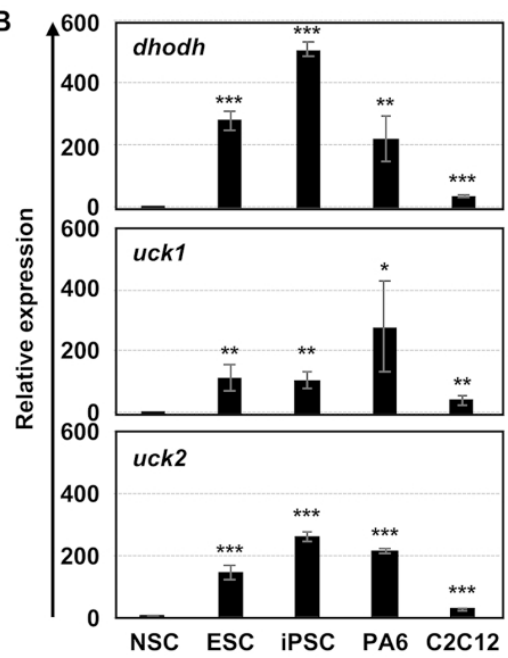

E

22

Supplementary Figure 5. Increased dhodh /uck1 expression ratio in PSCs $180 \times 230 \mathrm{~mm}(270 \times 270 \mathrm{DPI})$ 
A

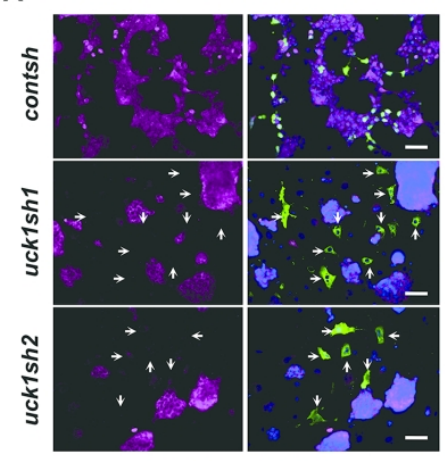

GFP/Uck1/DAPI

C
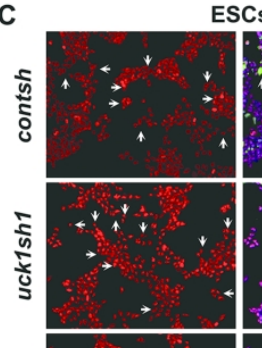

స్

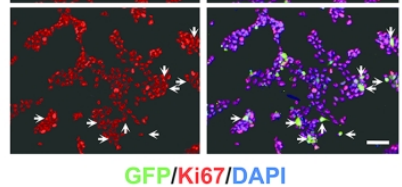

GFP/Ki67/DAPI

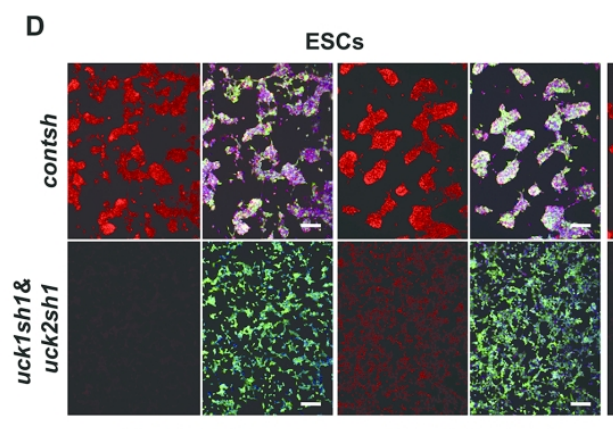

GFP/Uck1/DAPI

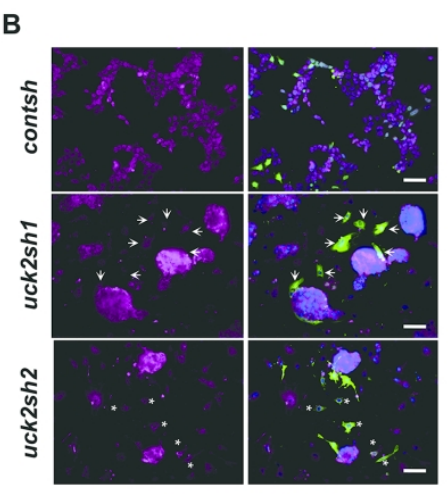

GFP/Uck2/DAPI

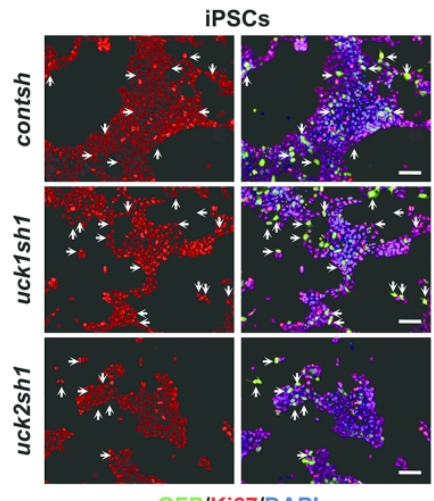

GFP/Ki67/DAPI

iPSCs

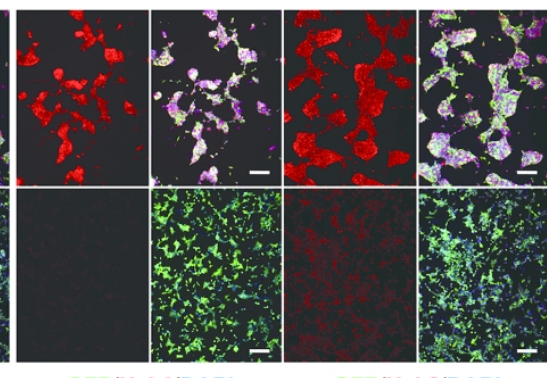

GFP/Uck1/DAPI

Supplementary Figure 6. Effects of uck1 and uck2 knockdown in PSCs $176 \times 240 \mathrm{~mm}(270 \times 270 \mathrm{DPI})$ 


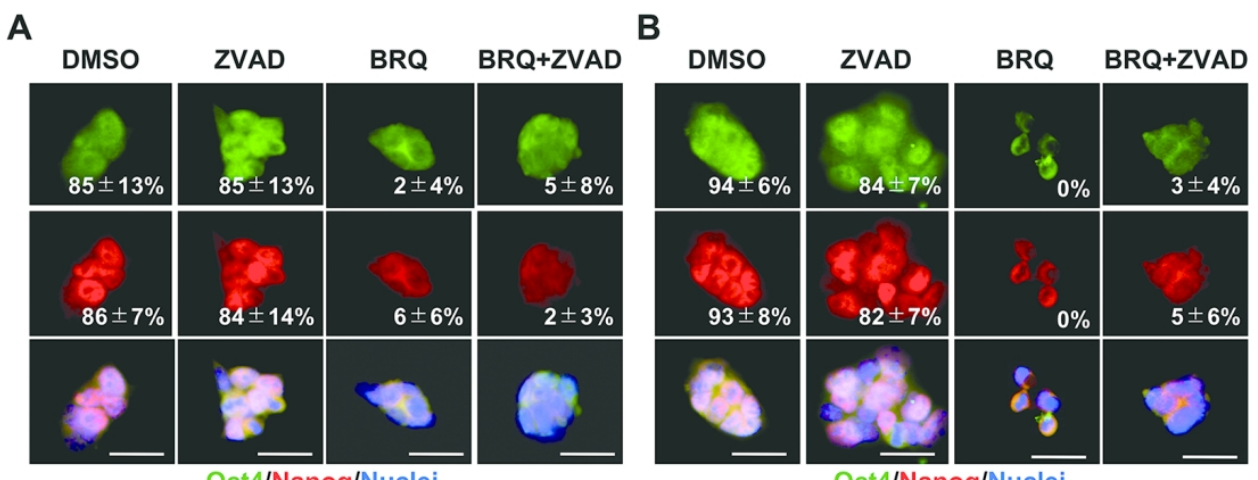

Oct4/Nanog/Nuclei

Oct4/Nanog/Nuclei

C

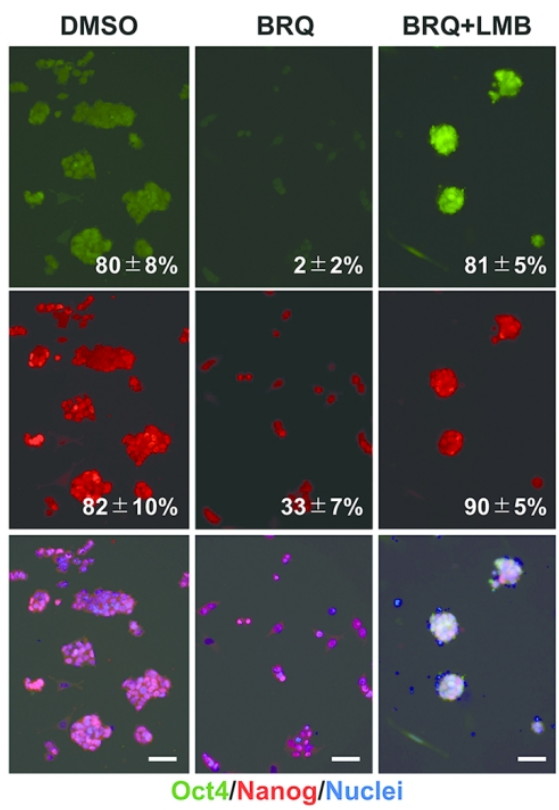

D

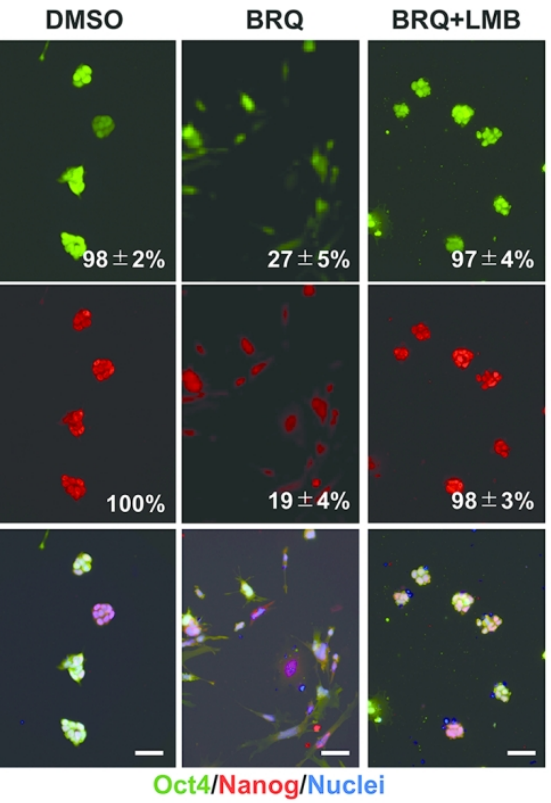

Supplementary Figure 7. LMB prevented the BRQ-dependent nuclear export of PSC markers $158 \times 166 \mathrm{~mm}(270 \times 270 \mathrm{DPI})$ 


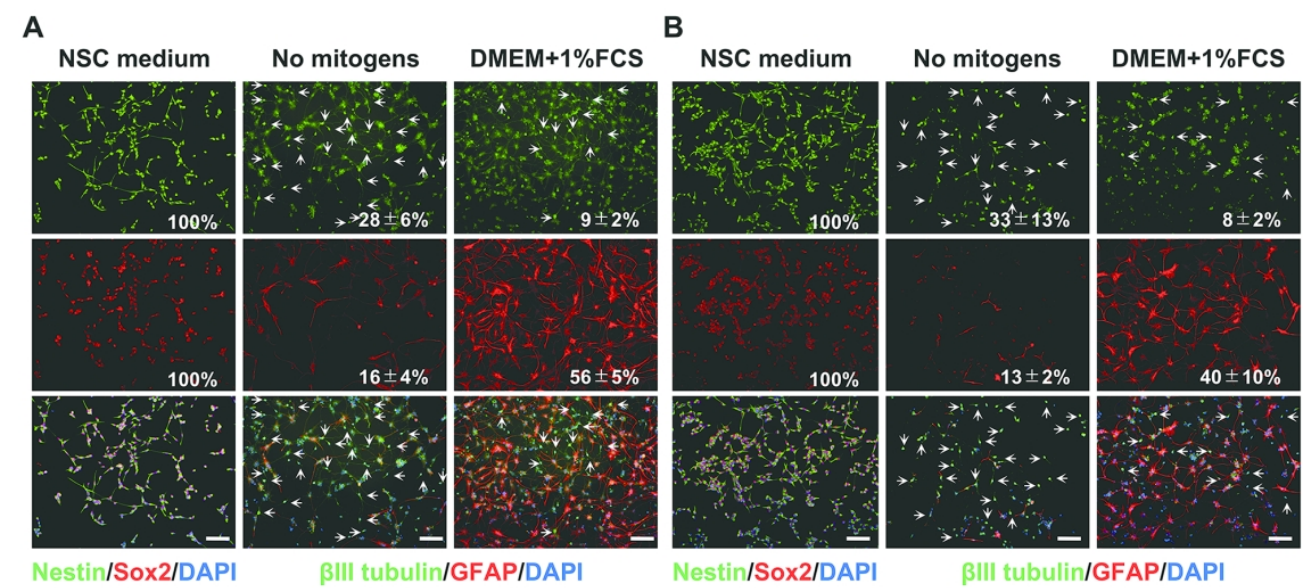

C

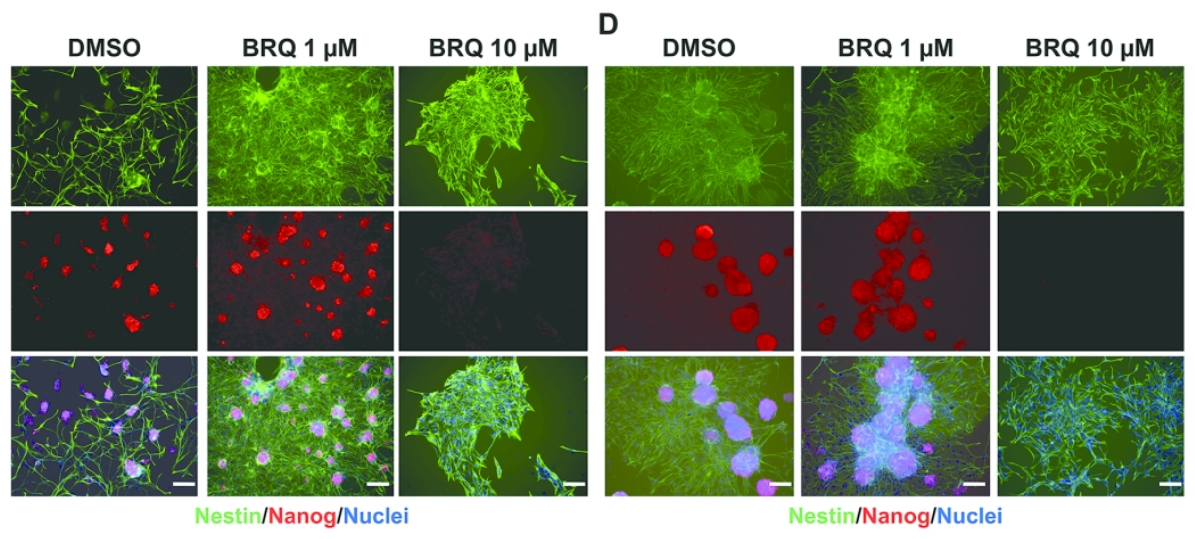

Supplementary Figure 8. PSC-derived NSC differentiation $187 \times 165 \mathrm{~mm}(270 \times 270 \mathrm{DPI})$ 


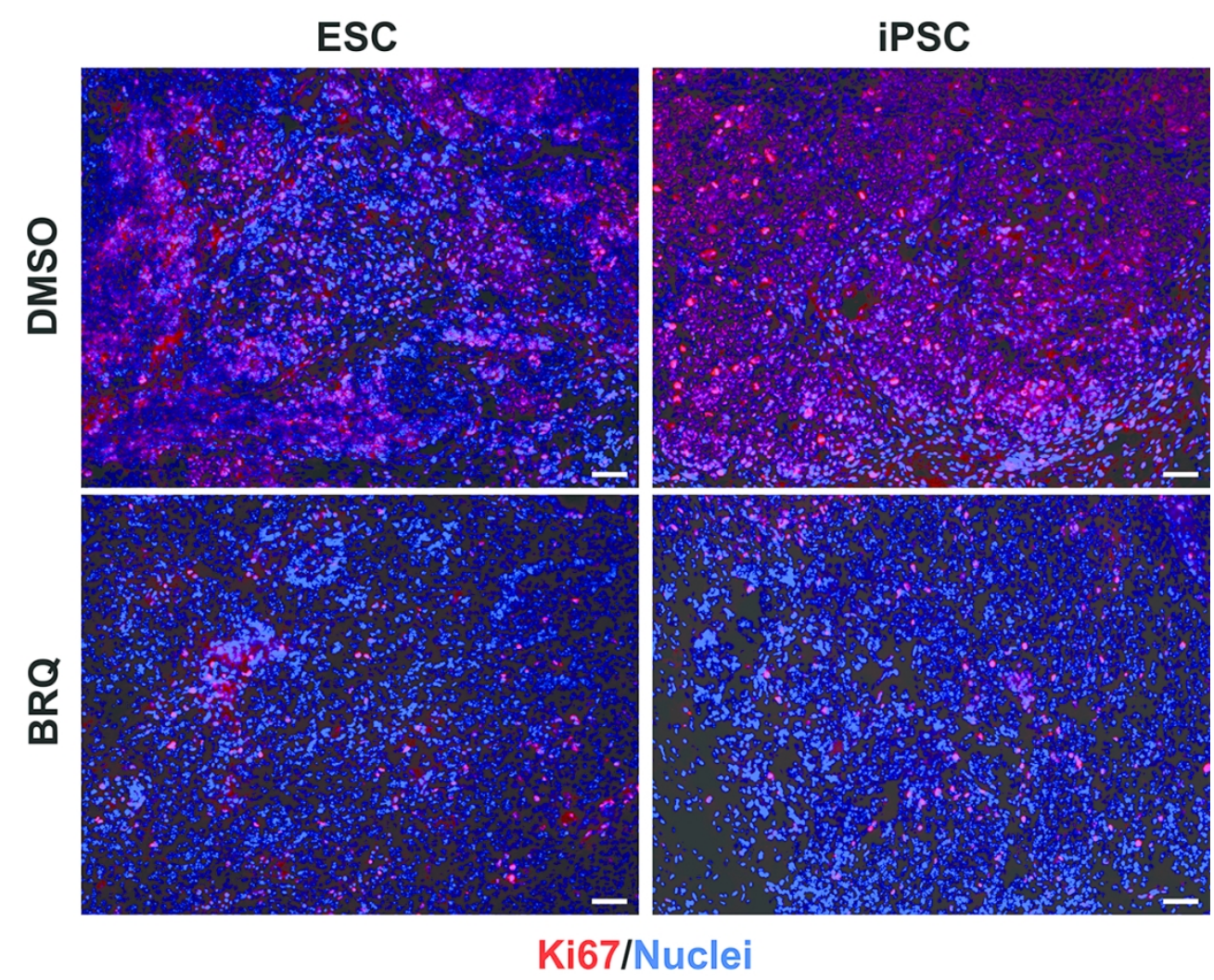

Supplementary Figure 9. BRQ administration decreased Ki67 + cells in teratomas $126 \times 105 \mathrm{~mm}(270 \times 270 \mathrm{DPI})$ 

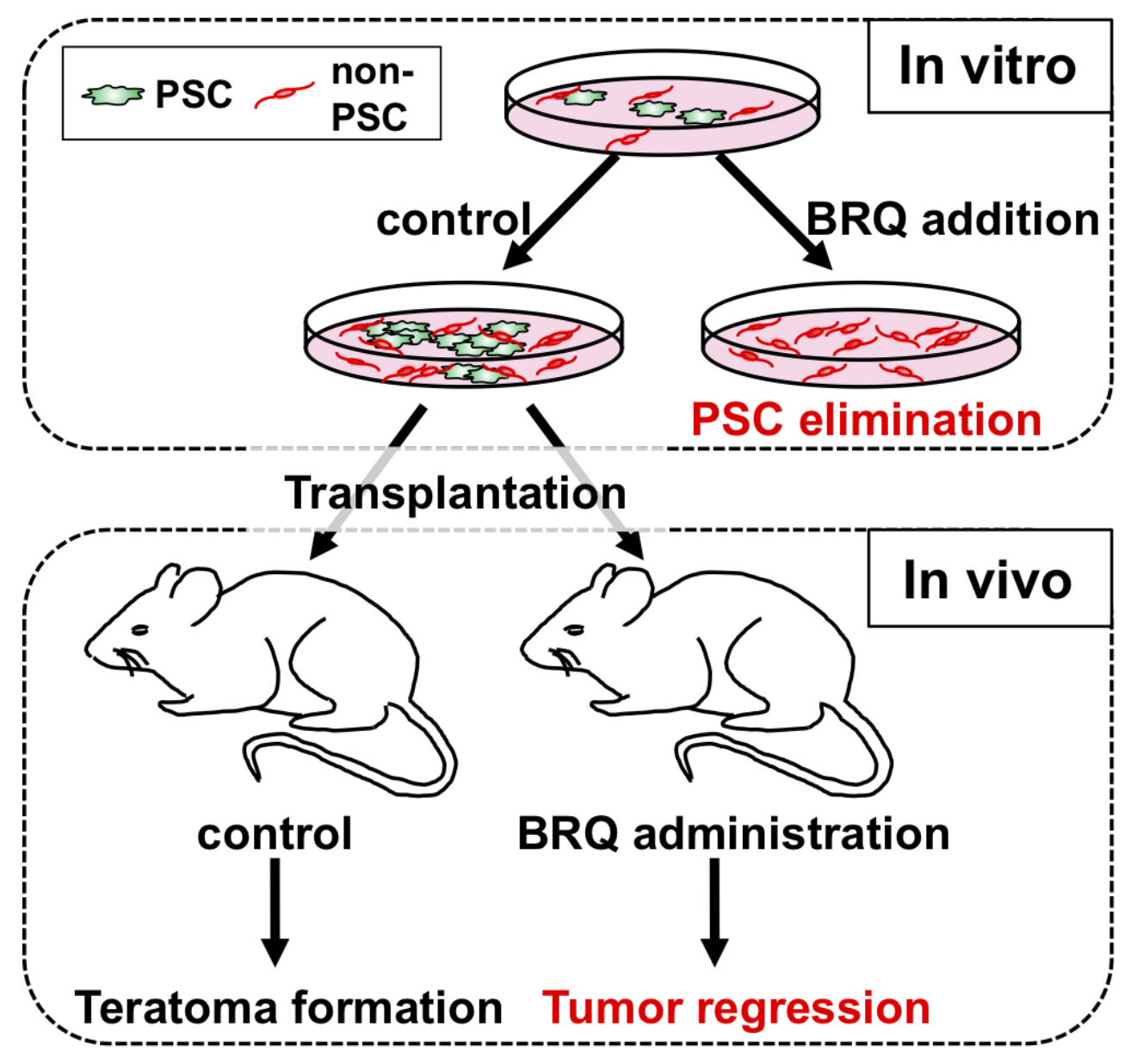

This study demonstrated that Brequinar (BRQ), an inhibitor for the dihydroorotate dehydrogenase (DHODH), not only induced cell cycle arrest and cell death in PSCs in vitro but also prevented tumorigenesis by selectively eliminating PSCs in vivo. Given that BRQ was less toxic for non-PSCs, these findings identified DHODH inhibitors, such as BRQ, can be used as fundamental chemicals for PSC-based cell therapy.

$102 \times 95 \mathrm{~mm}(254 \times 254 \mathrm{DPI})$ 